\title{
Regulation of Kinesin-3 Activity by Active Zone Protein SYD-2
}

\author{
Dissertation \\ for the award of the degree \\ “Doctor rerum naturalium” \\ Division of Mathematics and Natural Sciences \\ of the Georg-August-University Göttingen
}

submitted by

Sailaja Mandalapu

born in Tenali

Göttingen 2011 

I hereby declare that the presented PhD thesis "Regulation of Kinesin-3 activity by active zone protein SYD-2" has been written independently and with no other sources or aids than quoted.

Göttingen 10.11.2011

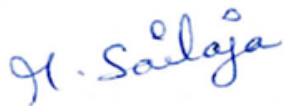

Sailaja Mandalapu 


\section{Table of contents}

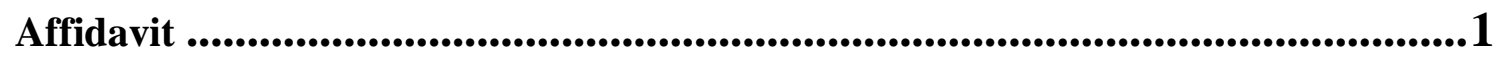

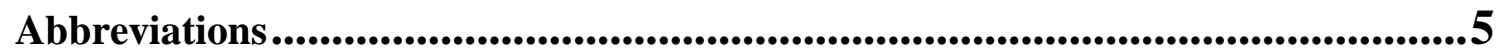

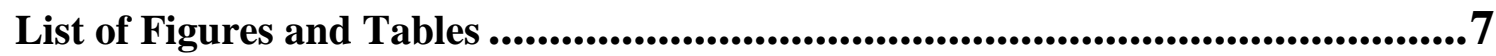

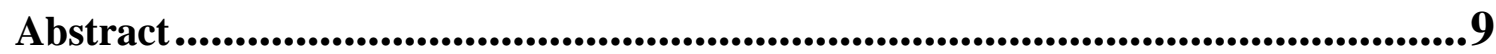

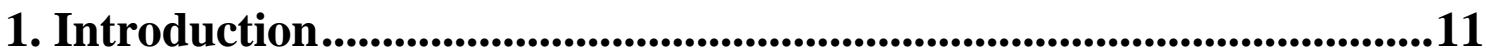

1.1 Kinesins 13

1.1.1 Structure of kinesins 13

1.1.2 Structure of UNC-104 15

$\begin{array}{ll}\text { 1.1.3 Classification } & 17\end{array}$

1.1.4 Role of Kinesins in neuronal transport 19

1.1.5 Role of UNC-104 in neuronal transport 20

1.1.5a Role of UNC-104 in axonal transport 20

1.1.5b Role of UNC-104 in the dendrites 22

1.1.6 Processivity of Kinesin-3 motors 23

1.1.7 Regulation of KIF1A/UNC-104 25

1.1.8 Kinesins in disease $\quad 28$

1.2 The family of Scaffolding proteins: Liprins $\quad 29$

1.2.1 Structure of Liprin $\alpha /$ Syd-2 30

1.2.2 Classification of Liprins 32

1.2.3 Role of SYD-2 / Liprin- $\alpha$ in synaptogenesis 33

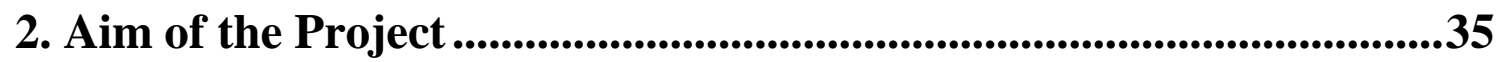

3. Materials and Methods..........................................................................38

3.1 Reagents 38

3.2 Plasmid isolation and Cloning $\quad 45$

3.3 Transformation 46

$\begin{array}{ll}3.4 \text { Recombinant protein expression and purification } & 47\end{array}$

3.4.1 Expression of UNC-104 constructs 47

3.4.2 Expression of SYD-2 constructs 49

3.5 Pull down assay 49

3.6 Western blotting 50

3.7 Microtubule gliding assay 51

3.7.1 Preparation of taxol stabilized microtubules 52

3.7.2 Preparation of flow chambers $\quad 52$ 
3.8 Single molecule assay by Total Internal Reflection Fluorescence microscopy 54

3.8.1 Principle of TIRF microscopy 54

3.8.2a Preparation of Diethylentriamin (DETA) cover slips 56

3.8.2b Provider's instruction to the use of plasma cleaner 57

3.8.3 Preparation of samples 58

3.8.4 Image acquisition and analysis 58

3.9 ATPase assay 59

3.10 Blue Native Poly Acryl amide Gel Electrophoresis (PAGE) 60

3.10.1 Principle of BN PAGE $\quad 60$

$\begin{array}{ll}3.10 .2 \text { Preparation of gel } & 61\end{array}$

3.10.3 Preparation of the sample $\quad 61$

3.10.4 Electrophoresis 62

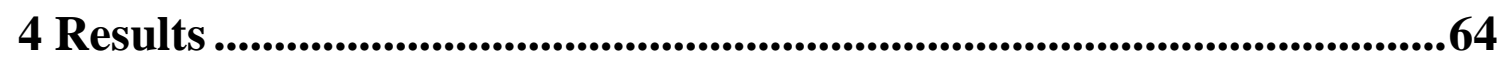

4.1 Truncated UNC-104 and SYD-2 proteins interact in vitro. 64

4.2 FHA domain is essential for faster UNC-104 movement. 69

4.3 SYD-2 positively regulates UNC-104 activity 72

4.4 SYD-2 regulates UNC-104 activity in a single molecule assay
by TIRF microscopy

4.5 SYD-2 decreases the catalysis rate of UNC-104 82

4.6 Increase in velocity is not due to formation of UNC-104 dimers 84

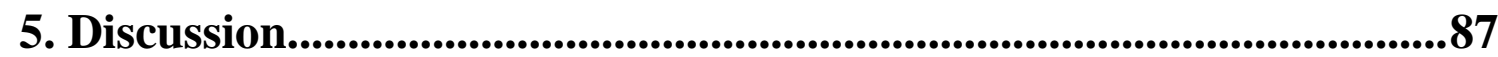

5.1 UNC-104 interacts with SYD-2 in vitro $\quad 88$

$\begin{array}{ll}\text { 5.2 FHA domain is important for faster motility } & 89\end{array}$

5.3 SYD-2 enhances UNC-104 velocity in FHA containing UNC-104 constructs 90

5.4 SYD-2 enhances UNC-104 velocity in the single molecule assay 91

5.5 SYD-2 decreases the ATPase activity of UNC-104 motor. 93

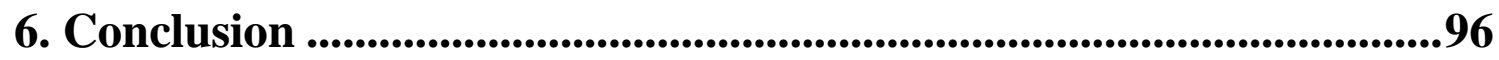

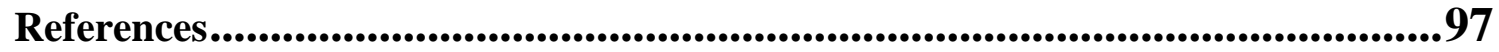

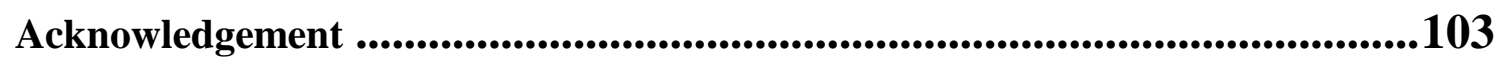

Curriculum Vitae ......................................................................................................105 


\section{Abbreviations}

aа

ADP

AMP

AMPA

ATP

AZ

BN

BSA

CC

C. elegans

cDNA

C-terminus

DDH2O

DETA

DTT

DMSO

DNA

dNTP

EDTA

EGTA

FHA

GFP

GTP

GRIP

His

HRP

IFT

IgG

IPTG

KCL

KD

KH2PO4

$\mathrm{KHz}$

KHC

KIF

KLC

$\mathrm{KOH}$

LAR amino acid

Adenosine-5'-DiPhosphate

Ampicillin

$\alpha$-amino-3 hydroxy-5-methyl1-4-isoxazoleproprionic acid

Adenosine-5'-TriPhosphate

Active Zone

Blue Native

Bovine serum albumin

Coiled Coil

Caenorhabditis elegans

complementary DNA

Carboxy-terminus

Double distilled water

Diethylenetriamine

Dithiothreitol

Dimethylsulfoxide

Deoxyribonucleic acid

deoxyNucleotide TriPhosphate

Ethylene Diamine Tetra acetic Acid

Ethylene Glycol Tetra acetic Acid

Fork Head Associated

Green Fluorescent Protein

Guanosine-5'-TriPhosphate

Glutamate Receptor Interacting Protein

Histidine

Horse Radish Peroxidase

Intra Flagellar Transport

Immunoglobulin $\mathrm{G}$

Isopropyl $\beta$-D-1-thiogalactopyranoside

Potassium chloride

Kilo Dalton

Potassium di hydrogen phosphate

Kilohertz

Kinesin Heavy Chain

Kinesin Superfamily protein

Kinesin Light Chain

Potassium Hydroxide

Leukocyte antigen related 


\begin{tabular}{ll} 
LB & Luria-Bertani \\
LDH & Lactate Dehydrogenase \\
LH & Liprin Homology \\
Liprin & LAR interacting protein \\
max & Maximum \\
MgCl $_{2}$ & Magnesium Chloride \\
MBP & Maltose Binding protein \\
MHz & Megahertz \\
mM & milli Molar \\
$\mu$ M & Micro Molar \\
$\mu 1$ & Micro liter \\
Mpa & Mega Pascal \\
MT & Microtubules \\
NADH & Nicotinamide Adenine Di nucleotide Phosphate \\
Na2HPO4 & Disodium Hydrogen Phosphate \\
NaCl & Sodium chloride \\
Ni & Nickel \\
nm & nanometer \\
N-terminal & Amino-terminal \\
NMDA & N-methyl-D-Aspartate \\
OD & Optical Density \\
P12 & PIPES 12 \\
PAGE & Polyacrylamide Gel Electrophoresis \\
PBS & Phosphate-Buffered Saline \\
PCR & Polymerase Chain Reaction \\
PH & Pleckstrin Homology \\
PIPES & Piperazine-N,N'-bis(2-ethanesulfonic acid) \\
PIP2 & Phosphatidylinositol 4,5-bisPhosphate \\
PK & Pyruvate Kinase \\
RIM & Rab3 Interacting Molecule \\
rpm & revolutions per minute \\
RT & Room temperature \\
SAM & Sterile Alpha Motif \\
SDS & Sodium Dodecyl Sulfate \\
syd/SYD & synapse defective \\
TBS & Tris Buffer Saline \\
TBST & TBS Tween triton-X buffer \\
U & Units \\
unc/UNC & un-coordinated \\
wt & Wild type \\
& \\
\hline &
\end{tabular}




\section{List of Figures and Tables}

\section{Introduction}

Figure 1 Structure of kinesin-1 14

Figure 2 Structure of UNC-104 and its proposed dimer confirmation 16

$\begin{array}{lll}\text { Figure } 3 & \text { Phylogenetic tree of the kinesin superfamily } & 18\end{array}$

Figure 4 Kinesins involved in neuronal transport and their cargo 19

Figure 5 Tracks of C. elegans wt and mutant on a bacterial lawn 21

Figure 6 Structure of syd-2 and its homologs in D. melanogaster and human 31

\section{Methods}

Figure 7 Schematic diagram of the flow chamber used for in vitro motility assays 53

Figure 8 Schematic diagram depicting the principle of TIRF 55

\section{Results}

Figure 9A Yeast 2 Hybrid results from Wagner et al, 2009

Figure 9B Schematic representation of the SYD-2 \& UNC-104 domains used in the study

Figure 10 Pull downs of UNC constructs

Figure 11 Gliding assay of UNC-104 construct U653-GFP-His6 71

Figure 12 Effect of SYD-2 on the velocity of UNC proteins in gliding assay: $\quad 74$

Figure 13 Representative kymographs of U653 in a single molecule analysis $\quad 78$

Figure 14 Representative kymographs of U800 in a single molecule analysis $\quad 79$

Figure 15 Effect of SYD-2 on the velocity of UNC proteins in single molecule assay 80

Figure 16 BN PAGE gel image stained with Coomassie blue

\section{Tables}

Table 1 Interacting partners and functional importance of UNC-104/KIF1A domains 16

Table 2 Kinesin superfamily members and their cargo linked to diseases 29

Table 3 Functional domains of Liprin- $\alpha$ and their interacting partners 32

Table 4 Motor velocities in gliding assays $\quad 75$

Table 5 Motility properties of the UNC constructs in single molecule assay 81

Table 6 Enzymatic characterization of UNC-104 constructs 83 


\section{Abstract}

Kinesin is a large family of molecular motors that carry out various intracellular cargo transport processes along microtubules at the expense of ATP. UNC-104 (uncoordinated) is a C. elegans ortholog of mouse kinesin KIF1A known to be involved in long range transport of presynaptic vesicles towards synapses. Mutation in the unc104 gene impairs the anterograde transport of synaptic vesicles resulting in disrupted

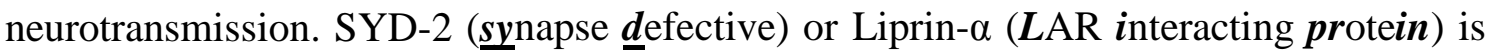
an UNC-104 interacting protein and loss of function mutations in this gene causes the synaptic vesicle mislocalization phenotype similar to unc-104 mutants. Previous work from our group showed the first evidence of the regulatory effect of SYD-2 on UNC104 in vivo but the underlying mechanism of this functional interaction is still unknown. We utilized the knowledge gained from our in vivo data to look at the effect of SYD-2 on the mechanochemical properties of UNC-104 to elucidate the underlying mechanism. In this study using biochemical and fluorescence-based activity assays we show that the FHA domain of UNC-104 is important for the motor to attain high motility rates and for processive movement in single molecule assays. SYD-2 interaction with the FHA 
domain of UNC-104 resulted in faster and longer run lengths. However analysis of the enzymatic properties of the motor in the presence of SYD-2 showed a decrease in the ATP hydrolysis rate and increased microtubule affinity, which needs further investigation for a valid explanation. 


\section{Introduction}

Intracellular transport of vesicles and membranes is essential for morphogenesis and normal functioning of the cell. Synthesized proteins and lipids are transported to their destination as membranous organelles or protein complexes. The trafficking of proteins is a tightly regulated process. Defects in trafficking are known to be a major cause of many diseases. Two classes of molecular motors Dyneins and Kinesins are known to carry out transport in neurons (Vale 2003). Motors move along microtubules using energy derived from ATP hydrolysis to generate force that can be used by the cell for

various ends, including transport of cargoes, segregation of organelles, destabilizing microtubules, alteration of morphology. Of these two, Kinesins represent a superfamily of molecular motors that are involved in the trafficking of various proteins, lipids and organelles to the destinations. Dyneins transport cargo towards the cell body in neurons (- end directed), while kinesins transport cargo towards the synapses (+ end directed). (Hirokawa 1998; Hirokawa et al, 2005). Kinesins play an important role in active transport especially in neurons. 
Neurons are highly polarized cells. Many are characterized by a single long axon and multiple shorter dendrites. Both the maintenance of and establishment of neuronal polarity involves coordinated and widespread regulation of the cytoskeleton and the membrane trafficking machinery.

Various membrane proteins like receptors, ion channels, transporters and adhesion molecules contribute to neuronal polarity. The polarized architecture is essential for neuronal function. Kinesins participate in the establishment and maintenance of neuronal polarity by selectively transporting various proteins and vesicles to either the axon or dendrites along microtubule tracks.

Kinesin was first characterized by Vale et al. in 1985 by using supernatants from axoplasm of giant squids to induce movement of microtubules along a cover slip and beads along microtubules (Vale 1985). Since then work from various groups has shed light on several aspects of kinesin characteristics, function and regulation. Till date 45 Kinesin superfamily proteins (KIFs) have been identified in mouse and human genome. Out of these 38 KIFs are expressed in the brain. UNC-104 is a C. elegans ortholog of mouse kinesin KIF1A that is expressed solely in neurons. It's a member of the Kinesin3 class of monomeric motors. 


\subsection{Kinesins}

\subsubsection{Structure of kinesins}

All KIFs share a common globular head/motor domain with 30-40\% identity. Outside the motor domain kinesins are quite divergent and subfamily specific. These divergent regions determine cargo binding and multimerization specific for each kinesin. The first to be characterized kinesin-1 is a tetrameric protein, consisting of two kinesin heavy chains (KHC) and two kinesin light chains (KLC) (Figure 1). The Kinesin heavy chain consists of 3 domains: N-terminal head/motor domain, $\alpha$-helical stalk domain and the Cterminal tail domain. The globular N-terminal head domain contains the ATP binding motif and a microtubule binding domain (Aizawa et. al 1992, Hirokawa et. al 1989) which is a common property of the family. The head domain is responsible for the movement empowered by the hydrolysis of ATP (Hirokawa 1989, Kikkawa et. al 2001, Nitta et. al 2004). It is attached via a 50 amino acid neck region to an extended $\alpha$-helical stalk which forms coiled coil (CC) structure upon dimerization with a second heavy chain. The neck region has been shown to be essential for the direction of motility (Endow \& Waligora 1998) or regulation of activity. It also interacts with the cargo that includes proteins, lipids and nucleic acids (Hirokawa 1989). The C- terminal tail domain is globular and interacts with the kinesin light chains (KLC). 


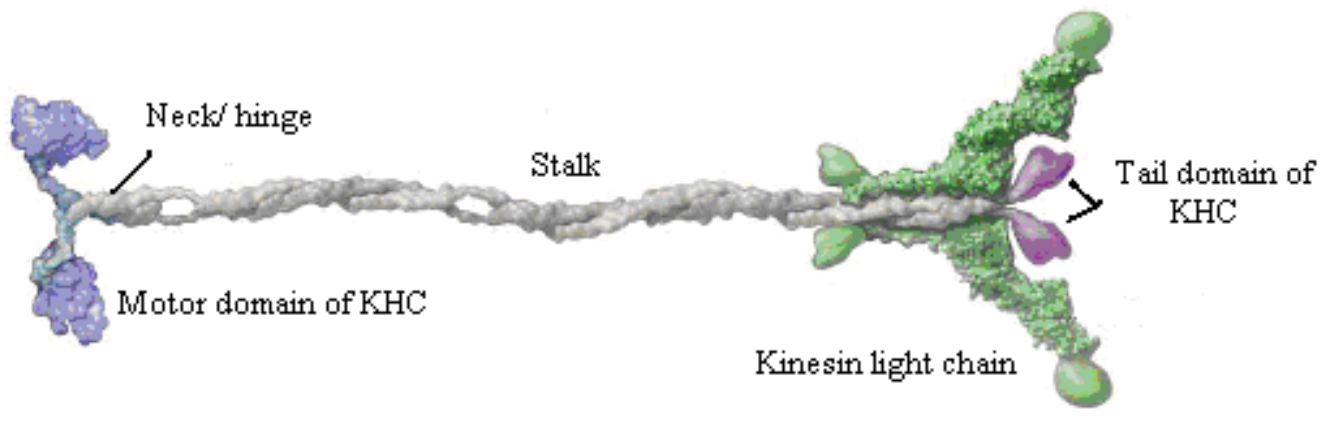

Figure 1. Structure of kinesin-1: kinesin-1 is a tetramer with the two heavy chains forming a dimer. The two light chains are represented in green. (adapted from Vale 2003). 


\subsubsection{Structure of UNC-104}

UNC-104 is an 180KD protein. Unlike Kinesin1, it is a monomer and its structure comprises of a motor domain (aa1-351), Coiled Coil (CC) 1 (aa429-462) fork head associated domain/FHA (aa488-604), CC2 (aa625-679) and a pleckstrin homology (PH) domain (FIG2). It has been predicted to form homodimers when concentrated in solution or on membranes, via the neck, adjacent to the motor domain (Tomishige 2002, Figure 2). Dimerization via the coiled- coiled domain allows the motor to move processively along microtubules like conventional kinesin (Tomishige 2002). The FHA domain has been shown to interact with the tail CC2 domain resulting in auto inhibition which has been proposed to be a regulatory mechanism (Lee et. al 2002). The PH domain binds to the membrane lipids of the cargo and clusters over the membrane (Klopfenstein et. al 2002). 


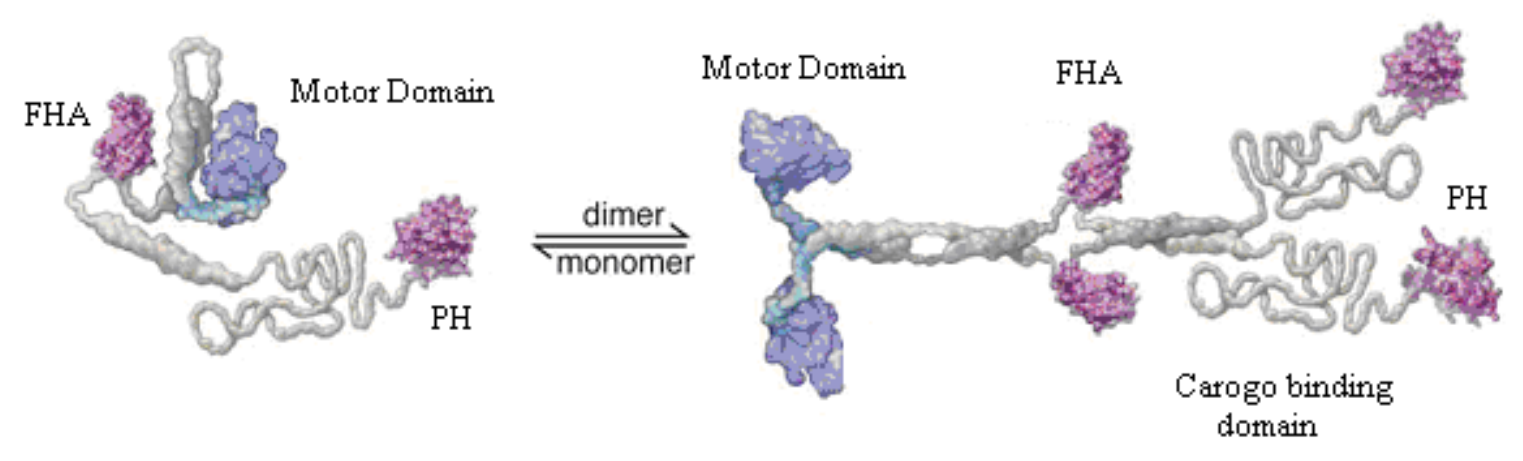

Figure 2. Structure of UNC-104 and its proposed dimer confirmation. UNC-104 is believed to form homodimers when functional and in high concentration. (adapted from Vale 2003).

\begin{tabular}{|c|c|c|c|}
\hline Protein & Domain / Motif & Binding Partner/ Cargo & Proposed function of interaction \\
\hline UNC-104/ & Motor domain & Microtubules & Walking Tracks \\
\hline \multirow[t]{7}{*}{ KIF1A } & & ATP & Energy for movement \\
\hline & Neck & $?$ & Helps in dimerization \\
\hline & FHA & its own coiled domain (Lee et al. 2004) & Self-regulation \\
\hline & Coiled-coil domain & Presynaptic vesicles & Synapse formation \\
\hline & & CC of UNC-104 (Tomishige et al 2004) & Processive movement \\
\hline & PH domain & Membrane lipids & Regulation \\
\hline & & (Klopfenstein et al 2002, 2004) & Membrane transport, \\
\hline
\end{tabular}

Table 1. Interacting partners and functional importance of UNC-104/KIF1A domains 


\subsubsection{Classification}

A complete search of the entire human genome using database search led to the identification of 45 KIFs using a database search, 38 of which were confirmed to be expressed (at the mRNA level) in brain through RT PCR or northern blotting (Miki et al 2001). Based on the primary structure around the motor domain, KIF's are classified into 3 subclasses (FIG3).

$\mathrm{N}$ - Kinesins: amino terminal motor domain

M - Kinesins: Middle motor domain

C - Kinesins: carboxy terminal motor domain

Out of the 45 KIFs 39 can be classified as N- kinesins while the rest belongs to the groups of M and C- kinesins. Of these 39 N-kinesins KIF1B and UNC-104/KIF1A are monomers. The N-kinesins are further divided into 11 classes and unc-104 belongs to the subclass of N-3 kinesins (Figure 3). All the N-Kinesins characterized so far are $\left(^{+}\right)$ end directed motors while C-Kinesins are (-) end directed motors. The M-Kinesins are involved in microtubule dynamics due to their depolymerizing properties. 


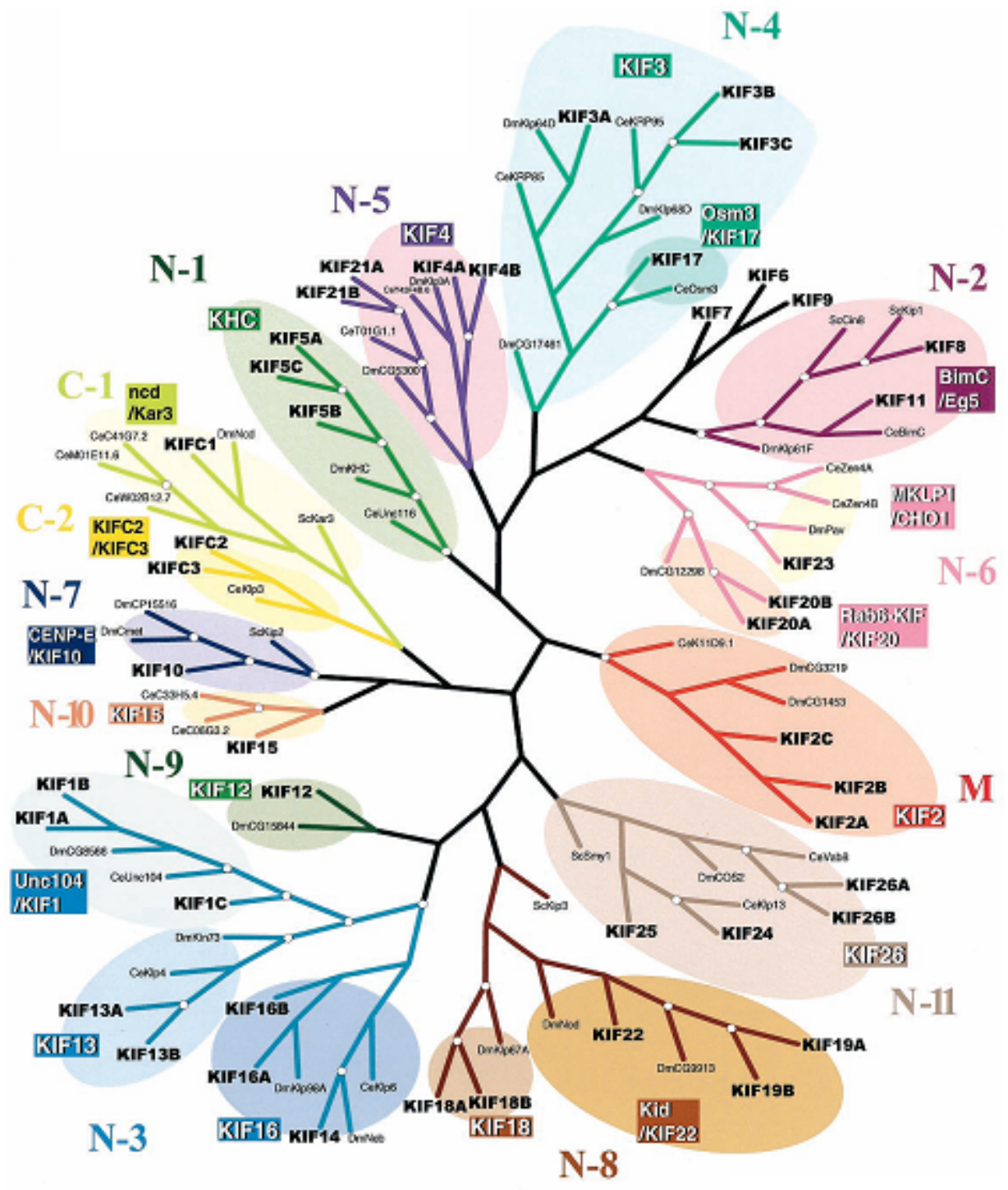

Figure 3. Phylogenetic tree of the kinesin superfamily. KIF1A and UNC-104 are the members of the kinesin sub family N3. (adapted from Miki et al 2001) 


\subsubsection{Role of Kinesins in neuronal transport}

Neurons are highly polarized cells. In dendrites microtubules are arranged bidirectionally with the microtubule $(+)$ end facing the end of the dendrite as well as towards the cell body. In the axons they are unidirectional with the $(+)$ end facing the synapse and the (-) end facing the cell body. In axons and dendrites various cargos are specifically transported by KIFs (Figure 4) along the microtubules. In the axons, precursors of synaptic vesicles are transported anterogradely (+ end) by KIF1A and KIF1B $\beta$.

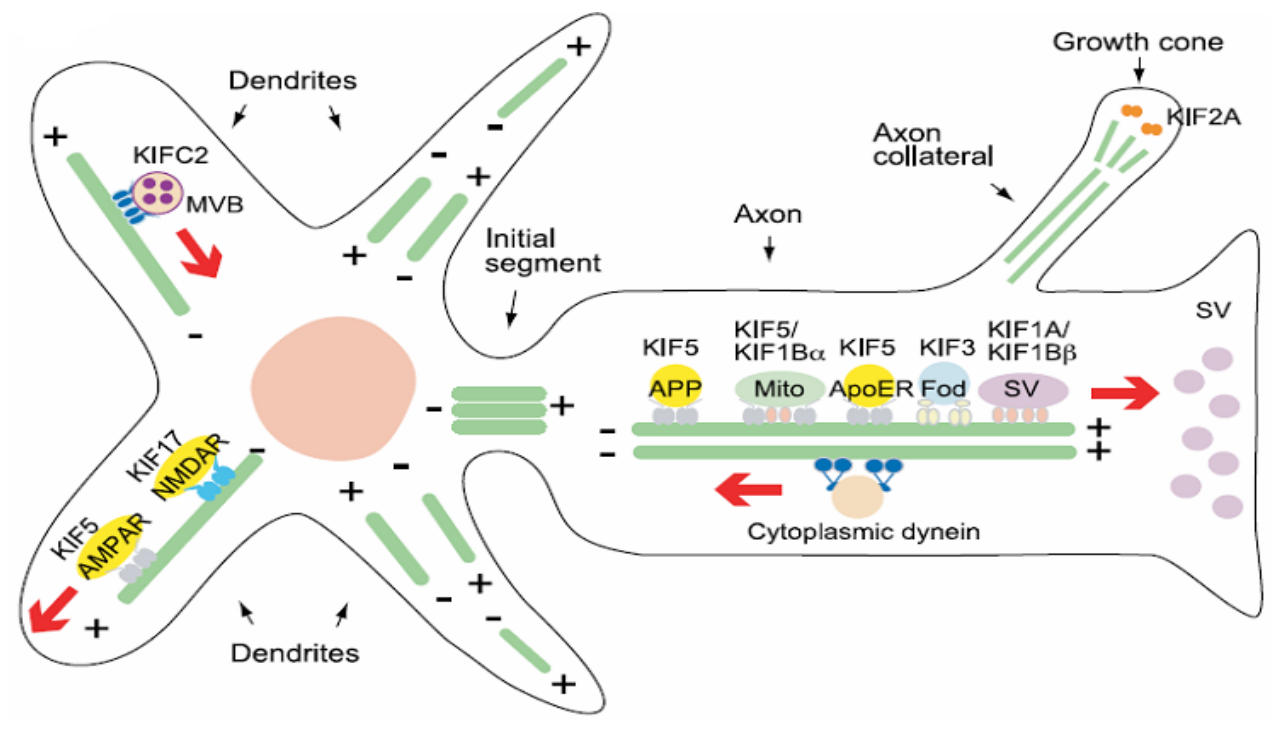

Figure 4. Kinesins involved in neuronal transport and their cargo. KIF1A/UNC-104 is involved in the transport of synaptic vesicles to the (+) end (towards the synapses). (adapted from Hirokawa \& Takemura review 2004) 


\subsubsection{Role of UNC-104 in neuronal transport}

\subsection{5a Role of UNC-104 in axonal transport}

In the axons KIF1A/UNC-104 plays a major role in the transport of synaptic vesicles and is important for neuronal differentiation and function. Knock out of mice kif1A showed motor and sensory nerve defects leading to death shortly after birth. The presynaptic termini had reduced number of synaptic vesicles while accumulation of synaptic vesicles was seen in the cell body (Yonekawa et al 1998). In C. elegans, unc104 mutation leads to slow and uncoordinated movement (FIG5, Hall \& Hedgecock 1991, Figure 5). The phenotype in unc mutants is similar to that seen in mice. They have reduced number of presynaptic vesicles localized at the synapses and accumulation of vesicles in the cell body (Hall and Hedgecock, 1991; Otsuka et al, 1991). The uncoordinated movement in unc-104 mutant worms is reminiscent of defective neuronal transmission. On the other hand they have normal neuronal anatomy and are viable. 
(A)

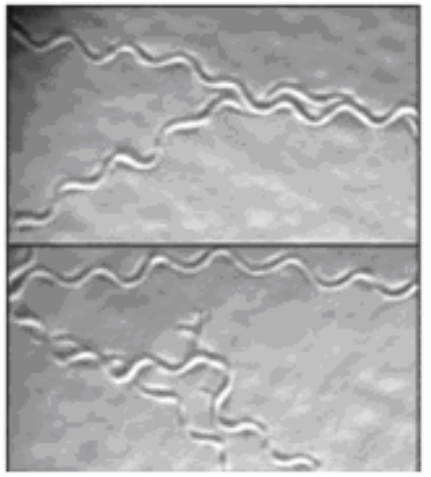

UNC-104 (wt)
(B)

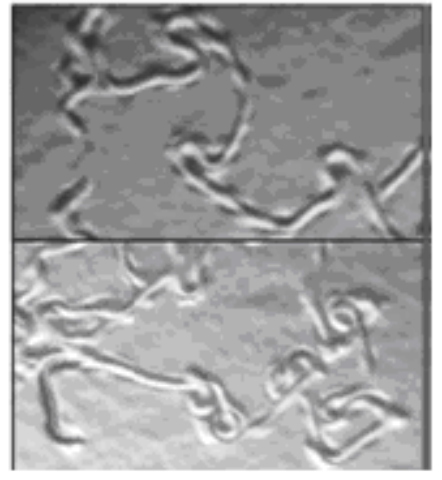

UNC-104 (e1265)

Figure 5. Tracks of C. elegans wt and mutants on a bacterial lawn. (A) Coordinated tracks of unc-104 wild type (B) Uncoordinated tracks of the mutants. (adapted from Hall \& Hedgecock 1999)

The kinesins are linked to their cargo via linker proteins which play key roles in synaptogenesis, active zone formation, signal transmission and neuronal development. Liprin- $\alpha /$ SYD-2 is an UNC-104 interacting protein and has been proposed to act as a linker to other active zone proteins that it interacts with. The interaction between the two was first reported by Shin et al through co-localization and co-immunoprecipitation experiments in rats (Serra-Pages et al, 1995). In immunoprecipitation experiments with KIF1A antibodies, two other Liprin- $\alpha$ associated proteins RIM and GIT1 were also pulled down. RIM/ Rab 3 interacting molecule is an active zone protein involved in neurotransmitter release and GIT1 is a multimodular scaffolding protein with an ADP ribosylation factor GTPase activating protein activity (Ko et al, 2003a). 


\subsection{5b Role of UNC-104 in the dendrites}

KIF1A co localizes with Liprin- $\alpha$ and GRIP (Glutamate Receptor Interacting Protein) in rat brain sections suggesting an important role in the clustering of the AMPA ( $\alpha$-amino3 hydroxy-5-methyl1-4-isoxazoleproprionic acid) receptors in the dendrites (Wyszynski et al, 2002). GRIP might act as an anchor for transporting AMPA receptors as well as other GRIP interacting proteins important for neurotransmission. Similar mechanism was shown for Kinesin 1 heavy chain where it interacts with GRIP-AMPA complex and targets the receptors to the dendrites (Setou et al, 2002). 


\subsubsection{Processivity of Kinesin-3 motors}

Processivity is a property attributed to the motor that is capable of taking several steps before dissociating from the microtubule. Conventional Kinesin/Kinesin-1, the first characterized motor takes $1008 \mathrm{~nm}$ steps before dissociating from the microtubules (Howard et al, 1989 \& Block et al, 1990). Kinesin 1 forms homodimers with the two motor heads and walks in a hand over hand mechanism, with the leading head always tightly bound to the microtubule, while the trailing head is weakly bound and leaps over the leading head resulting in $8 \mathrm{~nm}$ steps (Svoboda et al, 1993).

UNC-104/KIF1A is one of the fastest motor with an average velocity ranging from $1.0-$ $1.6 \mu \mathrm{m} / \mathrm{s}$ which is $2-3$ folds higher than that of Kinesin- 1 . The high processivity through a handover hand mechanism is ruled out since UNC-104/KIF1A is a monomer and does not have a second head to keep the motor still attached to the microtubule while taking the leap. Further studies with the different nucleotide states bound motor revealed an interaction between the K-Loop of Kinesin and E-Hook of the tubulin that keeps the motor bound to microtubules while the motor head is in transit thus achieving the $8 \mathrm{~nm}$

displacement. Though this one dimensional Brownian movement has been widely accepted UNC-104 does not seem to be solely dependant on this mechanism. Experimental evidences show that UNC-104 is capable of forming dimers at high concentration which could be a possible mechanism of fast axonal transport demands (Tomishige et al, 2002). In another study UNC-104 was shown to cluster on vesicles 
through the interaction of its $\mathrm{PH}$ domain with PIP2 on the vesicle membrane. This clustering was either speculated to lead to fast transport as a result of dimerization or cooperation among the multiple motors bound to the cargo (Klopfenstein et al, 2004). These studies point towards a mechanism that is unique to UNC-104 mediated transport. 


\subsubsection{Regulation of KIF1A/UNC-104}

A large proportion of cellular kinesins are not bound to cargo (Hollenbeck, 1989). This revelation led to the proposal that motors are enzymatically inactivated when not bound to cargo to curb the futile ATP consumption while being readily available for transport in the cytoplasm.

The first evidence of a probable tail - head inhibition was shown by Friedman \& Vale (1999). A series of experiments with various mutations in the neck region and truncated tail of conventional kinesin were conducted to determine the mechanism. In the ATPase assays the full length kinesin showed decreased ATPase activity than the tail deletion construct but in the gliding assay where the motors were bound to cover slips (mimicking cargo binding) there was no significant difference in velocity for all the constructs suggesting that the tail region represses the motor activity (Friedman \& Vale, 1999). Single molecule assays with TIRF (Total Internal Reflection Fluorescence) microscopy showed that the full length motor had many more pauses than the tail deletion construct though the velocities were comparable. These experiments point towards a self inhibitory mechanism involving the tail and the first hinge region thus rendering the non cargo bound kinesin inactive. The kinesin is believed to be in this inhibitory state until bound to its cargo and ready to deliver.

In yet another study Coy et al used ATPase assay and motility assays to study the tail mediated inhibition of Drosophila Kinesin heavy chain (KHC) activity and the effects of 
artificial cargo (silica beads). A seven fold increase in the rate of catalysis with the addition of beads was seen. The same catalysis rate was achieved with the deletion of the hinge region as when bound to the beads confirming that the tail mediated inhibition is indeed a regulatory mechanism for the kinesins and that cargo binding leads to the release of the inhibition.

The same mechanism was also shown to exist in the monomeric KIF1A motors as well. The Coiled coil (CC) domain in the stalk of KIF1A was shown to negatively regulate the motor activity upon binding to the FHA domain (Lee et al, 2004). The interacting proteins were proposed to relieve this inhibition upon binding to the motor.

In an other study the clustering of the UNC-104 motors on the lipid rafts resulted in high transport velocities thus suggesting that the clustering of the motor in the presence of cargo might in itself be a regulatory mechanism. The PH domain was shown to interact with the lipid rafts and was suggested that this clustering might trigger membrane transport (Klopfenstein et al, 2002).

These findings related to regulation were also shown to be a determining factor for processivity. It sure makes sense that negative regulation leads to loss of processivity. As different kinesins are involved in transporting diverse cargoes each kinesin adapts different regulatory mechanism. In the case of KIF1A or UNC-104, the regulatory mechanism seemed to be dependant on the type of cargo. When bound to vesicles its 
concentration seemed to play a role in increased processivity. On the other hand when bound to the active zone scaffolding protein SYD-2 the regulation could be the result of clustering of motors due to the multimerizing property of SYD-2 or the release of auto inhibition on binding of SYD-2 to the FHA domain of UNC-104. 


\subsubsection{Kinesins in disease}

Kinesins are involved in various intracellular transports and any disruption in their function may lead to disturbed transport of the cargo to the destinations. Disrupted transport in the axons as in the case of KIF1 and KIF5 can lead to blocks in the axons. Protein aggregates in neurodegenerative disease like Alzheimer's has been linked to defects in of amyloid precursor protein (APP) KIF5 transport (kamal et al, 2000 \& 2001). In the neurons KIF1A mediates the fast axonal transport of synaptic vesicles and is essential for the viability, maintenance and function of neurons (Yonekawa et al, 1998). Defects in Intra flagellar transport (IFT) in mice has been reported to give rise to several physiological defects including left right asymmetry defects, death and polycystic ovarian disease (Table 2.). In some neurodegenerative diseases, such as senile dementia, neuronal cell death could be caused by defects in the transport of synaptic vesicle precursors by KIF1A. 


\begin{tabular}{llll}
\hline Motor & Scaffolding proteins & Cargo & Disease \\
& & & \\
KIF1A & Liprin- $\alpha$ & AMPA receptor & Senile dementia (?) \\
KIF1B $\alpha$ & PSD-95 & Synaptic vesicles & Amyotrophic lateral sclerosis (ALS) \\
KIF1B $\beta$ & $?$ & $?$ & $?$ \\
KIF1C & sy-3-3 & synaptic vesicle & Charcot-Marie-Tooth-2A \\
KIF3 & KAP3 & Fodrin-associating vesicles & Left-right body axis determination, \\
KIF5 & GRIP & & Polycystic Kidney Disease \\
KIF17 & JIPs & AMPA receptor & ? \\
& & APP & Alzheimer's disease ?
\end{tabular}

Table 2. Kinesin superfamily members and their cargo linked to diseases. Modified version of Seog et al, 2004.

\subsection{The family of Scaffolding proteins: Liprins}

Synaptogenesis is a very complex and organized process which involves perfect alignment of the pre and post synaptic sites for an efficient neurotransmission. Studies over the years have been unfolding various molecules that contribute to synaptogenesis. 
Several molecules are involved on both sides of the synapse, the mechanism of which is poorly understood. Recent studies have been helpful in understanding the mechanisms in bits and pieces. Liprin or $\underline{\text { LAR }}$ interacting protein $\underline{\text { related protein }}$ is a family of multidomain proteins involved in synaptogenesis. Liprin- $\alpha 1$ belongs to the Liprin- $\alpha$ subfamily. It was originally isolated as a binding partner of the LAR (Leukocytecommon Antigen Related) receptor protein tyrosine phosphatase (Serra-Pages et al, 1995). Syd-2 (ynapse Defective) is a C. elegans ortholog of mammalian Liprin- $\alpha 1$, the only isoform found in C. elegans. It was isolated in a screen for mutants affecting localization of the synaptic vesicle protein synaptobrevin (Zhen \& Jin, 1999). It is an active zone (AZ) scaffolding protein involved in the presynaptic differentiation and post synaptic targeting of the AMPA receptors.

\subsubsection{Structure of Liprin $\alpha /$ Syd-2}

SYD-2 is a 130KD protein equipped with domains for protein-protein interactions. The structural motifs in SYD-2 molecule is composed of an N-terminal coiled-coils and the C-terminal SAM (ㅌterile alpha motif) domains (Figure 6). SYD-2 shares overall 50\% identity with human Liprin- $\alpha 1$. The C-terminal SAM domains are highly conserved 5070 amino acid structures that bind to proteins containing the SAM domains and lipid membranes. One common feature of all Liprins is that they are highly conserved within a 250 amino acids region in the C-terminus termed the Liprin Homology domain (LH). A span of 34aa in this region was conserved in all human and C. elegans Liprins known 
and a span of 85 amino acids are conserved in 6 out of 8 Liprins identified (Serra-Pages, 1995) suggesting a conservative role for the LH domain

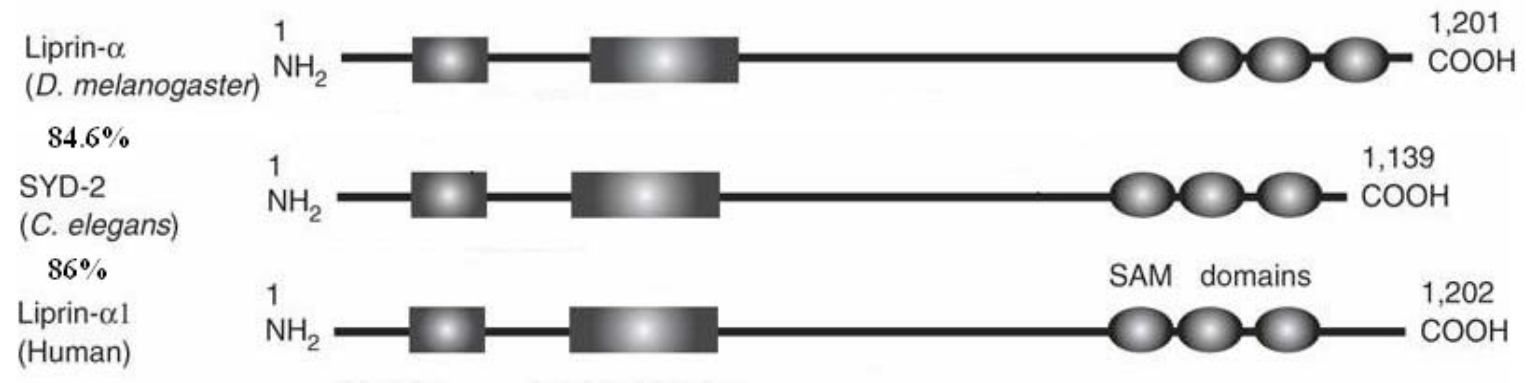

Figure 6. Structure of syd-2 and its homologs in D. melanogaster and human. SAM domains are highly conserved among species. The given similarity is for the SAM domains. (Modified from Dai et. al 2006)

The C-terminus LH domain binds to LAR and KIF1A in mammals. The coiled-coil domain has been shown to interact with many other active zone scaffolding proteins (Table 3) that in turn bind to other active zone components. They can form homodimers with the same subfamily through the N-terminal coiled- coil domains and heterodimers with the other Subfamily through the C-terminus. (Serra-Pages et al, 1995). 


\begin{tabular}{|c|c|c|c|}
\hline Protein & Domain / Motif & Binding Partner (reference) & Proposed function of interaction \\
\hline Liprin $\alpha /$ & $\mathrm{N}$ terminal CC region & RIM (Schoh et al. 2002, 18) & Scaffolding \\
\hline \multirow[t]{9}{*}{ SYD-2 } & & ELKS/ CASK (Ko et al. 2003b, 19) & Scaffolding \\
\hline & & GIT (Ko et al. 2003a, 20) & Membrane trafficking \\
\hline & & Kif1A (motor) (Shin et al. 2003, 21) & Transport \\
\hline & & Multimerization (29) & protein clustering at $\mathrm{AZ}$ \\
\hline & SAM domains & LAR (Serra-Pages et al. 1998, 22 ) & Receptor anchoring \\
\hline & & Liprin $\beta$ (Serra-Pages et al 1998, 22) & \\
\hline & & UNC-104 (Wagner et al 2009, 36) & Regulation \\
\hline & & CASK (Olsen et al. 2005, 23) & \\
\hline & PDZ binding motif & GRIP (Wyszynski et al. 2002, 24) & Receptor clustering \& transport \\
\hline
\end{tabular}

Table 3. Functional domains of Liprin- $\alpha$ and their interacting partners.

\subsubsection{Classification of Liprins}

Liprins are evolutionarily conserved proteins first identified as the binding partner to LAR Based on the sequence homology and binding properties Liprins are classified into $\alpha$ - Liprins and $\beta$ - Liprins. In human four known isoforms of $\alpha$ - Liprins $(\alpha 1-4)$ and 2 isoforms of $\beta$ - Liprins ( $\beta 1 \& 2$ ) exist while in C. elegans only one $\alpha$ - Liprin and $\beta$ - 
Liprin exist. They are highly conserved among themselves as well as with other subfamily. Among species SAM domains are the highly conserved regions.

\subsubsection{Role of SYD-2 / Liprin- $\alpha$ in synaptogenesis}

Synaptogenesis is a complex and poorly understood process which involves proper alignment of the presynaptic and post synaptic components. Liprin- $\alpha$ is one such molecule that was initially identified as an interacting partner to LAR (22), an adhesion molecule that is important for axon guidance and R7 photoreceptor targeting in drosophila and C. elegans. Liprin- $\alpha$ targeting of photoreceptor R7 axons to the correct layer in the medulla was reported to be both LAR dependant and independent (Kaufman et al, 2002 ; Hofmeyer et al, 2006). Several studies suggest a role for Liprin- $\alpha$ in localization. In Drosophila Liprin- $\alpha$ binding to Kinesin 1 was shown to be important for proper localization of synaptic vesicle proteins.

In C. elegans Liprin- $\alpha / \mathrm{SYD}-2$ plays various roles at the synapses. It is important for the localization of synaptic vesicle proteins synaptobrevin and synaptotagmin in an UNC104 dependant transport (Zhen \& Jin, 1999), active zone morphogenesis through association with 2 active zone proteins RIM and ELKS/CAST (Yeh et al, 2005 ; Dai et al, 2006) in neurotransmitter release by forming a ternary complex with CASK, Veli and MINT (Olsen et al, 2005). This ternary complex occurs on both sides of the synapse. Post-synaptically Veli/MALS bind to N-methyl-D-Aspartate (NMDA) receptors and was proposed to transport them to post synaptic membranes. Liprin- $\alpha$ is also important 
for targeting of AMPA receptors to the post synaptic sites by associating with Glutamate Receptor Interacting Protein (GRIP) and Kinesin 1 (Wyszynski et al, 2002).

In C. elegans Liprin- $\alpha /$ SYD-2 is also important for vulva development. It is important for the presynaptic organization in the egg laying synapses in HSNL neuron (Patel et al. 2006). In this study SYD-2 was shown to act downstream to synapse specificity molecule SYG-1 along with the scaffolding molecule SYD-1.

The various roles of SYD-2 and its association with KIF1A/ UNC-104 suggests that it acts as an adapter molecule to transport these synaptic proteins through KIF1A/UNC104 and that it plays a major role pre and post synaptic maturation.

SYD-2 association with the C. elegans motor UNC-104 has been recently shown to be important for UNC-104 regulation (Wagner et al, 2009). In SYD-2 mutants the axonal transport via UNC-104 was affected with an increase in net retrograde transport (- end).

These multiple roles of SYD-2 suggest that it acts as one of the first players in pre synaptic organization and then recruits other scaffolding molecules. It transports other presynaptic proteins acting as an adapter and regulating UNC-104 motility. 


\section{Aim of the Project}

Long range transport of synaptic vesicles and proteins by the microtubule based motors is essential for an efficient neurotransmission. The cargo is selectively delivered to the destination by specific motors. UNC-104/KIF1A is a neuronal motor and transports synaptic vesicle precursors to the synapses (Hall \& Hedgecock, 1991; Zhen \& Jin 1999). Loss of function of UNC-104 leads to accumulation of synaptic vesicles in the neuronal cell body resulting in neurotransmission defects (Yonekawa et al, 1998). SYD-2 is an UNC-104 interacting protein important for synaptogenesis. SYD-2 mutants exhibit defects in synapse morphology and mislocalization of synaptic vesicle and active zone proteins (Zhen et al, 1999; Kaufman et al, 2002, Wagner et al, 2009). Based on these studies SYD-2 was proposed to act as an adapter molecule for the transport of the mislocalized proteins by UNC-104. Though the role of UNC-104 and SYD-2/ Liprin- $\alpha$ in axonal transport and synaptogenesis is the focus of extensive research, there is very little or no focus on the regulatory mechanism involving the two. 
KIF1A and SYD-2 along with GRIP were shown to localize AMPA receptors to the synapses. So we assume that SYD-2 plays an important role in synaptogenesis and active zone architecture by localizing the scaffolding proteins to the synapses via UNC104. Liprin $\alpha 1 \& 2$, MALS, CASK and MINT proteins were highly enriched in synaptosomal fraction when imunoprecipitated with MALS-3 and were shown to the responsible for neurotransmitter release (Olsen et al, 2005). Probably SYD-2 acts as an adaptor molecule for these proteins and thus triggers the anterograde processive movement upon binding to UNC-104.

Self regulation of KIF1A motor by its own tail domain was shown by Lee et. al in 2004 . The coiled coil2 (CC2) of KIF1A negatively regulated the motor binding to microtubules by binding to the FHA region close to the neck. The motor remained inactive in the folded state. The similar mechanism for self regulation was shown by Verhey's group in the Kinesin1 motor where the binding of the light chains to the motor heads lead to inactive motor (Cai et al, 2007).

Most recent and more direct evidence for the regulatory function of SYD-2 was reported by our group (Wagner et al, 2009) in C. elegans. In this study we reported that SYD-2 interacts with UNC-104 through multiple domains and that SYD-2 regulates UNC-104 activity in vivo. SYD-2 binds to the fragment containing the FHA and coiled coil domain of UNC-104 with high affinity and this domain is also involved in self regulation. So we hypothesize that the binding of SYD-2 might lead to conformational 
change that switches the folded UNC-104 into a linear active motor and thus trigger anterograde movement.

In syd-2 mutants UNC-104 showed reduced anterograde transport with altered physical properties like lower velocity, less anterograde/more retrograde movements and increased pauses which signal towards a likely regulatory mechanism involving SYD-2.

In this study we focused on deciphering the effect of SYD-2 interaction on the motility and mechanochemical properties of UNC-104. We used fluorescent tagged motor fragment (U446, U653 and U800) to test the effect of SYD-2 fragment (608-1089). The motility properties of the motors can be best studied in vitro by using recombinant motors fused to fluorescent proteins. Microtubules gliding assay and single molecule analysis by TIRF microscopy are well established techniques to study the motile properties of motors like velocity and processivity. In this study we aim to gain a more clear understanding of the importance of SYD-2 and UNC-104 interaction. 


\section{Materials and Methods}

\subsection{Reagents}

All the chemicals were purchased from ROTH Chemie other than the ones mentioned below.

\section{Chemicals}

Complete EDTA free protease inhibitor cocktail tableta Protein Marker Broad Range, Anti mouse igG peroxidase

Peroxidase conjugated Goat anti-mouse IgG Mouse Monoclonal anti-polyhistidine clone His-6

Mouse anti GFP antibody mouse anti RGS-his antibody Anti MBP-HRP monoclonal antibody syd2 cC-20 (C-term)antibody Donkey anti goat igG-HRP Sild-A-Lyzer 10 KD 3-12 ml 8 Stck Rosetta 2 (DE3) singles competent cells Modified Lowry Protein As 1 Kit ATP

\section{Company/Catalogue no}

Roche 04693132O9W

NEB P7702S

Sigma A4416-5ML

Sigma A4416-5ML

Sigma H1029- 2M

Roche 11814460001

Qiagen 34610

NEB E8038 S

Santa Cruz sc-15656

Santa Cruz sc-2020

Perbio science 66810

Novagen US170953-4

Thermo electron 0023240

Sigma Aldrich A7699-5G 
GTP 5' triphosphate sodium salt hydrate

Glucose oxidase Type VII Aspergillus niger

Catalase from bovine liver

Quick Load 1 kb DNA Ladder

Taq DNA Polymerase

Nu PAGE LDS sample loading buffer (4X)

DH5 alpha Competent E. coli (High Efficiency)

Albumin Fraktion V Proteasefrei

ECL Western Blotting Dection Reagenz 1000 cm2

Ni-NTA Superflow

Tubulin bovine brain

IPTG dioxanfrei

Hyperfilm ECL 18 x $25 \mathrm{~cm} 25$ sheets

HiTrap Q FF

NuPAGE® MOPS SDS Running Buffer

Amylose Resin $15 \mathrm{ml}$

Vivaspin 650.000 MWCO Polythersulfon Membrane

Artikels BigDye ${ }^{\circledR}$ Terminator v1.1 Cycle Sequencing Kit

Beta- NAD, reduced disodium salt

Taxol from Taxus Brevifolia 95\% g

(EGTA) Ethylene Glycol-Bis (B-Aminoethyl Ether)

Phospho(Enol) pyruvate

PK/LDH enzyme solution in 50 glycerol $\mathrm{L}$

Ligate-it rapid Lig. Kit (100 RCTN)
Sigma G8877-250mg

Sigma G2133-50KU221KU/G solid

Sigma C40-100mg132KU/mg protein

NEB N0468 S

NEB M0267 L

Invitrogen NP0007

NEB C2987 I

Roth T844.2

Amersham Biosciences RPN 2109

Qiagen 30430

Tebu Bio 027TL238-E

Roth 2316.4

Amersham RPN2103K

Amersham 17505301

Invitrogen NP000102

NEB E8021S

Viva science VS0631

Appled biosciences 4337450

Sigma N9535-15VL

Sigma T7402-\%m

Sigma E8145-50G

Sigma P7002-100mg

Sigma P0294-5M

USB Europe 784001 KT

\section{Medium}

\section{Luria-Bertani medium}

$\begin{array}{ll}\text { Tryptone } & 10 \mathrm{~g} \\ \text { Yeast extract } & 5 \mathrm{~g} \\ \text { Nacl } & 5 \mathrm{~g} \\ \text { Water } & \text { to } 1 \text { Litre }\end{array}$


SOC medium

$\begin{array}{ll}\text { Tryptone } & 20 \mathrm{~g} \\ \text { Yeast extract } & 5 \mathrm{~g} \\ \mathrm{NaCl} & 0.5 \mathrm{~g} \\ \mathrm{KCl} & 2.5 \mathrm{ml}(1 \mathrm{M} \text { stock }) \\ \text { Water } & \text { to } 970 \mathrm{ml}\end{array}$

Sterilize by autoclaving

Cool to $<50^{\circ} \mathrm{C}$ add

$\mathrm{MgCl}_{2}$

Glucose

\section{RICH medium}

$\begin{array}{ll}\text { Tryptone } & 10 \mathrm{~g} \\ \text { Yeast extract } & 5 \mathrm{~g} \\ \mathrm{NaCl} & 5 \mathrm{~g} \\ \text { Glucose } & 2 \mathrm{~g} \\ \text { water } & \text { to } 1 \mathrm{~L}\end{array}$

\section{TPM medium}

$\begin{array}{ll}\text { Peptone } & 20 \mathrm{~g} \\ \text { Yeast extract } & 15 \mathrm{~g} \\ \mathrm{NaCl} & 8 \mathrm{~g} \\ \mathrm{Na} 2 \mathrm{HPO} 4 & 2 \mathrm{~g} \\ \text { KH2PO4 } & 1 \mathrm{~g}\end{array}$

Add DDH2O to

950ml. adjust $\mathrm{pH}$ to

7.0 with $\mathrm{KOH}$ and autoclave

\section{Buffers and stocks for Western Blotting}


20X MOPS electrophoresis buffer

$\begin{array}{ll}\text { MOPS } & 104.6 \mathrm{~g} \\ \text { Tris } & 60.6 \mathrm{~g} \\ \text { SDS } & 10 \mathrm{~g} \\ \text { EDTA } & 3 g \mathrm{~m}\end{array}$

to $1 \mathrm{~L}$ with water

\section{M DTT}

770mg Dithiothreitol (DTT, MW 154) in $10 \mathrm{ml} \mathrm{H2O.} \mathrm{Store} \mathrm{at} \mathrm{-20c} \mathrm{as} \mathrm{1ml}$ stocks.

\section{Coomassie blue}

Coomassie blue $\quad 0.5 \mathrm{~g}$

Methanol 200ml

Acetic acid $\quad 50 \mathrm{ml}$

$\mathrm{DDH}_{2} \mathrm{O}$ to $500 \mathrm{ml}$

\section{Ponceau S}

$\begin{array}{ll}0.50 \% & \text { Ponceau S } \\ 1 \% & \text { acetic acid }\end{array}$

\section{Blotting Buffer}

$25 \mathrm{mM}$

$192 \mathrm{mM}$

$20 \% \mathrm{v} / \mathrm{v}$

$\mathrm{DdH}_{2} \mathrm{O}$ to 1Litre
Tris

Glycine

Methanol 3.03g

$14.4 \mathrm{~g}$

200ml

PBS-Puffer pH 7,4 1000 ml

$\begin{array}{ll}\mathrm{KCl} & 0,2 \mathrm{~g} \\ \mathrm{KH} 2 \mathrm{PO} 4 & 0,2 \mathrm{~g}\end{array}$




$\begin{array}{ll}\mathrm{Na} 2 \mathrm{HPO} 4 & 1,15 \mathrm{~g} \\ \mathrm{NaCl} & 8 \mathrm{~g}\end{array}$

TBS Buffer (Wash Buffer) pH 7.5 1L

$\begin{array}{lll}10 \mathrm{mM} & \text { Tris } & 1.21 \mathrm{~g} \\ 150 \mathrm{mM} & \mathrm{NaCl} & 8.76 \mathrm{~g}\end{array}$

TBS/Tween/Triton Buffer (Wash buffer) pH 7.5 1L

$20 \mathrm{mM} \quad$ Tris $2.42 \mathrm{~g}$

$500 \mathrm{mM} \quad \mathrm{NaCl} 29.2 \mathrm{~g}$

$0,05 \% \mathrm{v} / \mathrm{v} \quad$ Tween-20 500ml

$0,2 \% \mathrm{v} / \mathrm{v} \quad$ Triton $\mathrm{X}-100 \quad 2 \mathrm{ml}$

Blocking solutions

$3 \% \quad$ BSA

$5 \% \quad$ Milk

in TBS/PBS buffer

Buffers and stocks for protein purification

1 M IPTG

IPTG $\quad 4,76 \mathrm{~g}$

water $20 \mathrm{ml}$

$1 \mathrm{ml}$ aliquotes stored at $-20 \mathrm{oC}$

pMal Columm buffer pH 7,4 1000 ml

20mM Tris 2,42 g

$200 \mathrm{mM} \mathrm{NaCl} \quad 11,7 \mathrm{~g}$

$1 \mathrm{mM}$ EDTA $\quad 2 \mathrm{ml}$ of $0.5 \mathrm{M}$ stock 
DdH2O to $1 \mathrm{~L}$

Ni lysis buffer pH 8

$\begin{array}{ll}\mathrm{NaH} 2 \mathrm{PO} 4 & 3.45 \mathrm{~g} \\ \mathrm{NaCl} & 7.31 \mathrm{~g} \\ \text { Imidazole } & 0.68 \mathrm{~g}\end{array}$

DdH2O to $500 \mathrm{ml}$

Ni wash buffer pH 6.0

$\mathrm{NaH} 2 \mathrm{PO} 4.45 \mathrm{~g}$

$\mathrm{NaCl} \quad 7.31 \mathrm{~g}$

$\mathrm{MgCl} 2 \quad$ add $1 \mathrm{ml} 1 \mathrm{M} \mathrm{MgCl} 2$ stock (23.8g I 25ml ddH2O)

DdH2O to $500 \mathrm{ml}$

Ni elution buffer pH 7.2

$\begin{array}{ll}\mathrm{NaH} 2 \mathrm{PO} 4 & 3.45 \mathrm{~g} \\ \mathrm{NaCl} & 7.31 \mathrm{~g} \\ \text { Imidazole } & 17.02 \mathrm{~g} \\ \mathrm{MgCl} 2 & 1 \mathrm{ml} 1 \mathrm{M} \mathrm{MgCl} 2 \text { stock (23.8g I 25ml ddwater) } \\ & \\ \text { DdH2O to } 500 \mathrm{ml} & \end{array}$

Mono Q `A’ 500ml pH 6.8

$\begin{array}{ll}\text { PIPES } & 3.78 \mathrm{~g} \\ \mathrm{MgCl} 2 & 1 \mathrm{ml}(1 \mathrm{M} \text { stock }) \\ \text { EGTA } & 0.19\end{array}$

DdH2O to $500 \mathrm{ml}$ 
Mono Q 'B’ 500ml pH 6.8

PIPES $\quad 3.78 \mathrm{~g}$

$\mathrm{MgCl} 2 \quad 1 \mathrm{ml}$ (1M stock)

EGTA $\quad 0.19$

$\mathrm{NaCl} \quad 29.22 \mathrm{~g}$

DdH2O to $500 \mathrm{ml}$

Stocks and buffers for MT Gliding and TIRF Assay

ATP $100 \mathrm{mM}$ in water $\mathrm{pH} 7.0$

25mM GTP in P12 buffer

BRB80 buffer pH 6.8

80mM PIPES

$1 \mathrm{mM}$ EGTA

$1 \mathrm{mM} \mathrm{MgCl} 2$

P12 buffer pH6.8

12mM PIPES

$2 \mathrm{mM} \mathrm{MgCl} 2$

$1 \mathrm{mM}$ EGTA

1mM DTT

$\mathrm{pH}$ adjusted with $\mathrm{KOH}$

prepared as $10 \mathrm{x}$ stock and stored at $-20^{\circ} \mathrm{C}$

NADH/PEP mix

6mM NADH 20ul

30mM PEP 20ul

in 1X P12 buffer 


\section{PK/LDH enzyme mix}

$\begin{array}{ll}5.5 \mathrm{U} / \mathrm{ul} \mathrm{PK} & 20 u l \\ 2.0 \mathrm{u} / \mathrm{ul} \mathrm{LDH} & 20 u l \\ \text { 4M KCL } & 1 \mathrm{ul} \\ 1 \mathrm{X} \mathrm{P12} \mathrm{buffer} & 59 u l\end{array}$

\subsection{Plasmid isolation and Cloning}

All the unc-104 constructs were a gift from Vale's lab. All the constructs were amplified by PCR and cloned into pET 17b expression vector between Nde1 and Kpn1. GFP was cloned between Kpn1 and Xho1 in frame with the unc-104 followed by His 6 for affinity purification. The sequences were confirmed with restriction analysis and sequencing with T7 forward and reverse primers as well as insert specific primers in the lab. The MBP-SYD-2 C-terminus construct (608-1086) was cloned into pMAL C2x (NEB) between BamH1 and Xba1. The sequences were confirmed by restriction analysis and sequencing with malE Primer (NEB \#S1237S) and M13/pUC Sequencing Primers (NEB \# S1211S) as well as insert specific primers.

\section{List of primers used for cloning}

MAL-1089-XbaI 5’ ACTTCTAGACTAAGATCCAATCTGGAGTGCATATGC 3’

MAL-BamHI-608 5’ GAGGATCC TCACTG GCCGACGTCG GTCAATCG 3’

\section{List of primers used for sequencing}


T7 F 5' TAATACGACTCACTATAGGG 3'

T7 R 5’ CCGCTGAGCAATAACTAGC 3’

UNC-2153for 5' CATCTTTACTTGAATTCCCGG 3'

UNC-2380 R 5’ TTAAGTCTTCTCCTGGTGGC 3’

Ce-6375 F 5’ TGTTCT TACCACGACA TTTGC 3’

U653 Rev 5' GGCACAATCTTATATATCTG 3’

U653 For 5’ ATGTCATCGGTTAAAGTAGC 3’

Seq-syd-2-941 F 5’ CGCACCGATC AAGAAACTCG G 3’

E.coli cells carrying the appropriate plasmid (from a glycerol stock/ from transformations) were inoculated into $5 \mathrm{ml}$ LB medium containing suitable antibiotics, and cultured overnight at $37^{\circ} \mathrm{C}$ with agitation at $200 \mathrm{rpm}$. The culture was precipitated by centrifugation at $4000 \mathrm{rpm}$ for $10 \mathrm{~min}$. Plasmid isolation from the bacterial pellet was done using QIAprep Spin Miniprep kit (Qiagen), according to the specifications in the product manual. The isolated plasmid was resuspended in $\mathrm{ddH}_{2} \mathrm{O} /$ provided TE buffer

\subsection{Transformation}

Plasmids carrying the desired cDNA fragment were transformed into transformation competent E. coli DH5 $\alpha$ cells for propagation or the desired E. coli competent cells for expression. An aliquot of $50 \mu \mathrm{l}$ of these cells was thawed, mixed with $1-2 \mu$ l of plasmid DNA and cooled on ice for 30min. The cells were permeabilised by treating them to a heat shock. They were first incubated at $42^{\circ} \mathrm{C}$ for $45 \mathrm{sec}$, and then left on ice for $5 \mathrm{~min}$. To the transformed mixture of cells, $200 \mu \mathrm{l}$ SOC medium (without antibiotics) was 
added and cultured at $37^{\circ} \mathrm{C}$ for 1 hr with agitation. $100-200 \mu \mathrm{l}$ of this mixture was then plated on LB -selective antibiotic plates and incubated overnight at $37^{\circ} \mathrm{C}$. Individual colonies were subsequently picked and re-inoculated in 5ml LB (+antibiotic) medium, cultured overnight, and either used for plasmid isolation or protein expression.

\subsection{Recombinant protein expression and purification}

\subsubsection{Expression of UNC-104 constructs}

The UNC-104 constructs U653, U446 and U800 were transformed in Rosetta 2(DE3) single competent cells (Novagen, cat-71400-3) according to standard protocol mentioned above. 2L TPM culture was initiated with a $5 \mathrm{ml} \mathrm{o} / \mathrm{n}$ pre-culture and cultured at $37^{\circ} \mathrm{C}$ to an $\mathrm{OD} 0.6-0.8$ at $600 \mathrm{~nm}$. The cultures were let to cool down on ice for 20' and then induced with 500mM IPTG. The cultures were then grown o/n (12-16 hours) at $20^{\circ} \mathrm{C}$ for slow induction of the motor proteins. For the U800 construct the cells were grown at $8^{\circ} \mathrm{C}$ for 5 days since the protein yield was too low at $20^{\circ} \mathrm{C}$. Cells were harvested the next morning by centrifuging them in a GSA rotor at 4000rpm for 15'. The pellet was resuspended in Ni binding buffer and cells lysed by sonication with 15” interval after every 10” (45\% amplitude for $90 \mathrm{sec}$ ). The lysates were spun in an SS34 rotor at 12,000 rpm for 40 ' to remove cell debris. The lysates were applied to preequilibrated Ni NTA beads (Qiagen, cat-30410) and incubated for 1 hour on a rotating wheel $(10-20 \mathrm{rpm} / \mathrm{min})$ at $4^{\circ} \mathrm{C}$. The beads were then applied to $5 \mathrm{ml}$ polypropylene 
column (Qiagen), washed 3 times with 5 column volume of Ni NTA wash buffer. The proteins were eluted with a 500mM concentration of imidazole in wash buffer (pH7.2). The eluted protein was collected in $1 \mathrm{ml}$ fractions and was resolved by SDS PAGE on a $10 \%$ Bis-Tris gel (Invitrogen) to evaluate the fractions with protein. The fractions with high concentration of desired protein was dialyzed o/n against PIPES dialysis buffer (pH6.8) in a 3-12ml slide-A-Lyzer dialysis cassette (Pierce, Cat: 66810, MW:10.000) and subjected to second purification the next morning.

The dialyzed protein samples were applied to the FPLC for $2^{\text {nd }}$ purification using a HisTrap Q FF anion exchange column (Amersham, cat-17505301) using AKTA prime ${ }^{\mathrm{TM}}$ design (GE Healthcare). The column was equilibrated by washing once with Mono Q 'A' (wash buffer) followed by Mono Q 'B' (elution buffer) and then again with Mono Q 'A' buffer ( $1 \mathrm{ml} / \mathrm{min}$ flow rate and $<0.3$ Mpa pressure). The sample was loaded on to the column and washed with 5 column volumes of wash buffer. Continuous gradient elution was used to elute the sample using Mono Q 'A' buffer and Mono Q 'B' buffer (500mM Imidazole). The eluted fractions were selected from the highest peak obtained (approx 5-7 of $151 \mathrm{ml}$ fractions) and dialyzed o/n against PIPES dialysis buffer and concentrated to a volume of $200 \mu 1$ in a vivaspin 6 Polyethersulfone column (MWCO 50K, Sartorius, cat: VS0632). 50\% sucrose was added to a final concentration of $10 \%$ and snap frozen in liquid nitrogen. They were stored as $10 \mu \mathrm{l}$ aliquots at $-80^{\circ} \mathrm{C}$ until use. The protein concentrations were determined by Bradford using $0.5-2 \mathrm{mg} / \mathrm{ml} \mathrm{BSA}$ standards. 


\subsubsection{Expression of SYD-2 constructs}

The SYD-2 construct 608-1089 was transformed into TB1 chemically competent cells (section 4.2). Starter culture, large cultures and lysis were done according to standard protocol as mentioned in section (4.3.1). The only difference is that RICH medium was used to grow the $2 \mathrm{~L}$ cultures. The batch purification was as mentioned in section 4.3.1 except for the buffers. pMAL column buffer was used instead of Ni binding buffer and pMAL column buffer supplemented with $10 \mathrm{mM}$ Maltose was used for elution. The fractions were checked with SDS PAGE and the desired protein fractions dialyzed against PIPES dialysis buffer. The $2^{\text {nd }}$ purification elution and storing the protein were done as mentioned in section 4.3.1.

\subsection{Pull down assay}

$10 \mu \mathrm{L}$ of MBP-SYD-2 $(5 \mathrm{mg} / \mathrm{ml})(608-1089)$ and $10 \mu \mathrm{L}$ of $\mathrm{U}_{653}-\mathrm{GFP}-\mathrm{His} 6 / \mathrm{U}_{446}$-GFPHis6/U $\mathrm{U}_{800}-\mathrm{GFP}-\mathrm{His} 6(5 \mathrm{mg} / \mathrm{ml})$ were incubated on ice for 1 hour. $50 \mu \mathrm{L}$ of Amylose beads (NEB, cat-E8021S) prewashed thrice with MBP column buffer and blocked with $1 \%$ BSA (30' at RT) were added to all the samples and left for binding on a rotating wheel for I hour at $4^{\circ} \mathrm{C}$. For control U653/U446/U800 + MBP, U653/U446/U800 + beads were used. After the incubation the samples were centrifuged at $800 \mathrm{rpm}$ for 3 minutes and the supernatant were collected. The beads were washed twice with PBS and resuspended in $100 \mu \mathrm{L}$ sample buffer. $10 \mu \mathrm{L}$ of $1 \mathrm{x}$ sample buffer was added to the 
supernatant and were applied for SDS PAGE. MBP and UNC proteins were incubated as above to be processed as negative controls. After SDS PAGE the gels were subjected to western blotting with goat anti-SYD-2 C-term (1:1000Sigma) and mouse anti-His6 antibodies (1:1000Sigma). Donkey anti goat IgG HRP (1: 5000, Santa Cruz sc-15656) and anti mouse IgG HRP (1:2000, Sigma A4416-5ML)

\subsection{Western blotting}

$10 \mu \mathrm{L}$ of the beads and $20 \mu \mathrm{L}$ of the supernatants were resolved by SDS PAGE after boiling in the sample buffer for 10 minutes at $95^{\circ} \mathrm{C}$. A $1-2 \mathrm{~cm}-$ long stacking gel $(0.125 \mathrm{M}$ Tris-HCl pH6.8; 0.1\% SDS; 0.1\% APS; 0.1\% TEMED; 3.2\% acrylamide) and a 5cmlong resolving gel (0.375M Tris-HCl pH8.8; 0.1\% SDS; 0.05\% APS; 0.05\% TEMED; $10 \%$ acrylamide) was used. The samples were separated at $120 \mathrm{~V}$ in MOPS buffer and transferred to a nitrocellulose membrane (Roth, cat-4675.1) at a rate of $2.5 \mathrm{~mA} / \mathrm{cm}^{2}$ of the membrane for 1 hour in Tris-Glycine-methanol buffer. The broad range Protein Ladder (NEB, cat-P7702S) was used to estimate the size of separated proteins after staining with Ponceau S. The membranes were washed with PBS/TBS/TBST buffers respectively and incubated in blocking solution (5\%Milk /3\%BSA in TBS) for $1 \mathrm{hr}$ at RT followed by washes and incubation with the primary antibody at $4^{\circ} \mathrm{C}$ overnight. Membranes were then washed with PBS/TBS/TBST respectively, followed by incubation in HRP- conjugated secondary antibody for 1 hour at RT. The above incubation steps were accompanied by mild see saw agitation. The membranes were then washed with 
PBS/TBS/TBST respectively and treated with a chemiluminescent substrate using standard kits (Amersham ECL WB Detection kit, cat-RPN 2109) according to protocols described by the manufacturers. Finally, the signal was developed onto an X-ray film (hyper film ECL, Amersham, cat-RPN2103K) developed and fixed (Intas, Kodak).

\subsection{Microtubule gliding assay}

The Microtubule gliding assay employs the use of micro flow chambers which can accommodate small sample volumes (usually $5-10 \mu 1$ ). $5 \mu 1$ of UNC protein sample $(5 \mathrm{mg} / \mathrm{ml})$ was first applied to the flow channel on the cover slide and incubated in a humid chamber to prevent the sample from drying out. The motor proteins stick to the cover slip with their N-terminal tail domain. Antibodies can also be used to adsorb the motors to the cover slip but in our case the incubations work just fine without the use of antibodies. After 5 minutes of incubation the unbound motor is washed off with BRB80 buffer (80mM PIPES pH6.8, 1mM EGTA, $1 \mathrm{mM} \mathrm{MgCl}_{2}$ ). Then taxol stabilized microtubules diluted in an anti-bleach solution (BRB80 pH6.8, 1mM ATP, 2mM $\mathrm{MgCl}_{2}, 100 \mathrm{mM}$ glucose, $100 \mathrm{mM}$ DTT, $1 \mu \mathrm{g} / \mathrm{ml}$ Glucose Oxidase and $0.8 \mu \mathrm{g} / \mathrm{ml}$ Catalase.) is introduced into the chamber, left in humid chamber for few second (enough time for the microtubules to find the motor head) and then visualized with a inverted confocal microscope. The microtubules can be seen gliding over the motor lawn. 


\subsubsection{Preparation of taxol stabilized microtubules}

Rhodamine labeled tubulin and unlabelled tubulin were mixed at 1:10 ratio to a final concentration of $2 \mathrm{mg} / \mathrm{ml}$. The tubulin mixture was supplemented with $1 \mathrm{mM}$ GTP and $5 \%$ DMSO and incubated at $37^{\circ} \mathrm{C}$ for 30 minutes. $100 \mu 1$ of BRB80 buffer containing taxol to a final concentration of $10 \mu \mathrm{M}$ was added to the microtubules and incubated for an additional 5 minutes at $37^{\circ} \mathrm{C}$. The microtubules were then spun down through $40 \%$ glycerol cushion in a TLA 100.3 rotor for $10 \mathrm{~min}$ at $80,000 \mathrm{rpm}$ at $22^{\circ} \mathrm{C}$. The supernatant was discarded and the pellet was washed with BRB80 containing $10 \mu \mathrm{M}$ taxol. The supernatant was discarded and the pellet was resuspended in $100 \mu$ of BRB80 supplemented with $10 \mu \mathrm{M}$ taxol. The microtubules were stored at room temperature in dark until use.

\subsubsection{Preparation of flow chambers}

Micro flow chambers were prepared using a glass slide and a cover slip as shown in the FIG7. The double sticky tape (Tesa Germany) was stuck to the glass slide and flow cells (max 5) were cut from the tape. The cover slip was then placed over the tape and pressed to stick tightly. The flow chambers can accommodate 5-10 $\mu$ l of sample. The ends were sealed with glue after loading the sample to avoid drying of the sample while imaging. 


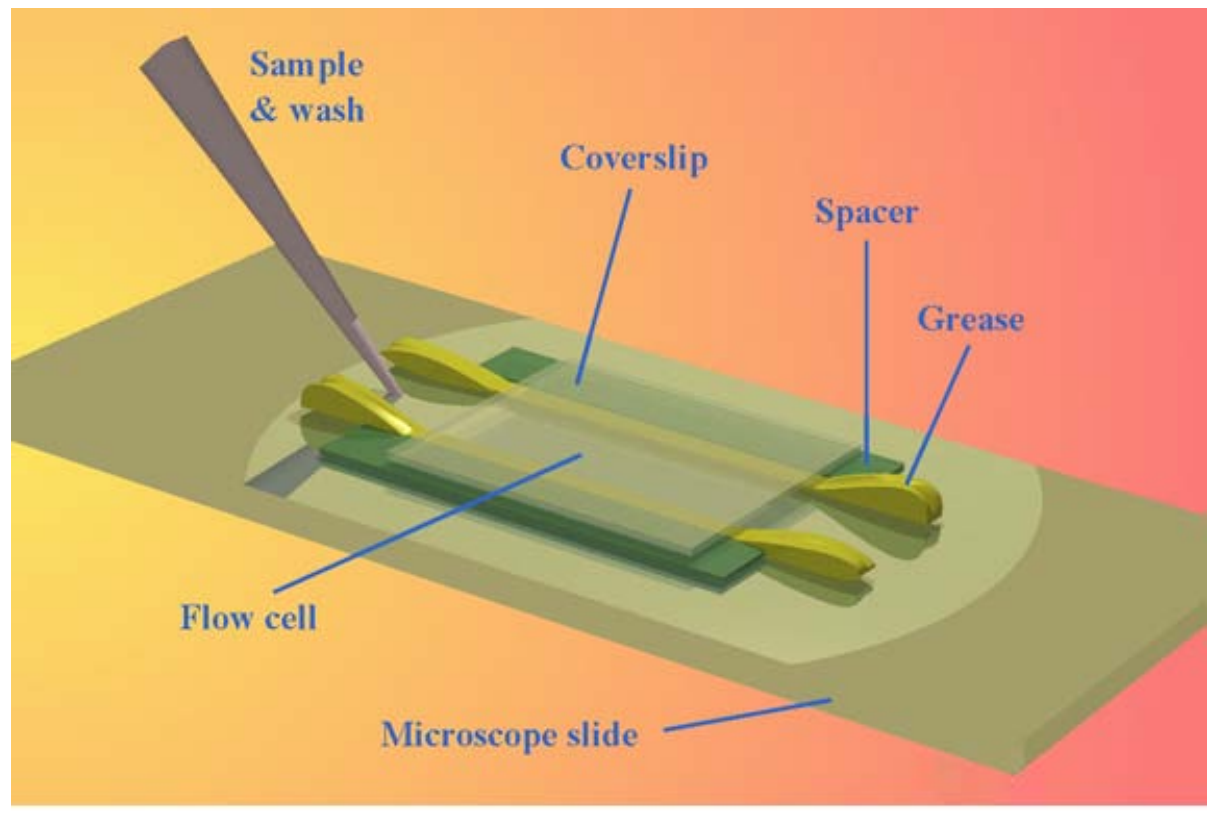

Figure 7. Schematic diagram of the flow chamber used for in vitro motility assays. Created by He Jiang, Laboratory of Molecular Cardiology, NHLBI, Bethesda (1997). 


\subsection{Single molecule assay by Total Internal Reflection}

\section{Fluorescence microscopy}

\subsubsection{Principle of TIRF microscopy}

Images acquired through conventional fluorescence microscopy are difficult to analyze because of high background fluorescence from other planes that are out of focus. Total internal fluorescence microscopy employs the phenomenon of total internal reflection which occurs at the interface between optically dense medium like glass and less optically dense medium like aqueous solution. At large angle of incidence, the excitation beam reflects back into glass and generates at the interface with water so called evanescent wave (FIG8). The evanescent wave has maximum of intensity at the surface and exponentially decays with the distance from the interface. Only molecules that are at the TIRF surface are excited and fluoresce, while molecules in the bulk of solution, at the distances larger than 100-200 nm are not excited and, respectively, do not fluoresce. TIRF efficiently rejects background signal from the bulk of solution and allows for super sensitive detection down to single molecules. 

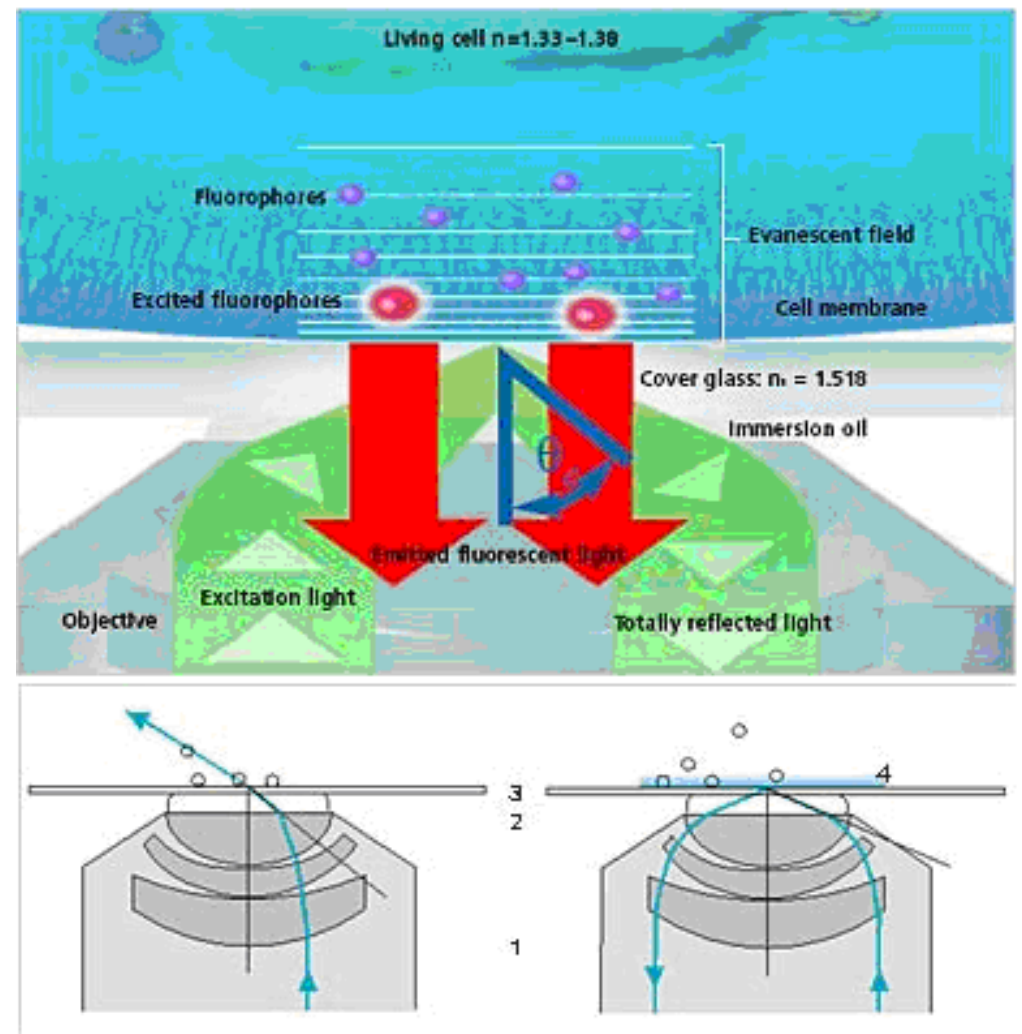

Beam path of incidence at less than the critical angle

Beam path of total reflection

Figure 8. Schematic diagram depicting the principle of TIRF.( adapted from Carl Zeiss) 


\subsection{2a Preparation of Diethylentriamin (DETA) cover slips}

The cover slips were cleaned in plasma cleaner (Harrick plasma, USA.) for 15 minutes. They were then placed in a container with deionized water and placed in an ultrasonic bath (Elmasonic, Elma, Switzerland). $200 \mu 1$ of Diethylenetriamine (DETA) was added to the water. After 5 minutes the water with DETA was exchanged with fresh deionized water and was incubated for another 5 minutes in the ultrasonic bath. This washing step was repeated thrice and then the cover slips were allowed to dry for an hour at $70^{\circ} \mathrm{C}$ in an oven. The cover slips are now ready to use. DETA is hydrophobic in nature and binds to the cover slips rendering a net -ve charge to the cover slips. The microtubules bind to the DETA and are thus immobilized which is important for single molecule assays. 


\subsection{2b Provider's instruction to the use of plasma cleaner}

Plasma cleaning involves the removal of impurities and contaminants from surfaces through the use of energetic plasma created from gaseous species. The plasma is created by using high frequency voltages (typically $\mathrm{kHz}$ to $>\mathrm{MHz}$ ) to ionize the low pressure gas. The sample is placed in the reaction chamber. Low flow rates (typically 5-10

SCFH) of process gas at low pressure (typically 200-600 mtorr) are subjected to RF (radio frequency) electromagnetic radiation at $8-12 \mathrm{MHz}$ creating plasma, at near ambient temperatures, within the chamber

When a gas under sufficiently low pressure is subjected to a high frequency oscillating electromagnetic field, the accelerated ions in the gas collide with the gas molecules ionizing them and forming plasma. The ionized gas particles in the plasma interact with solid surfaces placed in the same environment by:

\section{- Removing organic contamination from surfaces.}

The high energy plasma particles combine with the contaminant to form carbon dioxide or methane.

- Modifying or enhancing the physical and chemical characteristics of surfaces.

A chemical reaction occurs between the plasma gas molecules and the surface undergoing treatment. 


\subsubsection{Preparation of samples}

Single molecule analysis of motor by TIRF requires very low motor concentrations since at high motor density the molecules are so close that one can hardly differentiate between two individual GFP signals. The concentration was increased when very few events were seen in a given field probably due to less active motor. For all protein samples serial dilutions were made to a final concentration of 20nM - 50nM in BRB80 buffer. All the dilutions were made fresh for each imaging. The Motor constructs U446, U653 and U800 were incubated with SYD-2 on ice for 20 min prior to applying into the micro flow chamber for experiments with SYD-2.

\subsubsection{Image acquisition and analysis}

The images were acquired using a Zeiss Axiovert 200M microscope equipped with a Roper 512F iCCD camera (Visitron, Pucheim, Germany ) for gliding assays and a self built TIRF microscope. For gliding assays the image sequence was acquired at the rate of 1 frame/sec for $100 \mathrm{sec}$ and $0.1 \mathrm{sec}$ for $10 \mathrm{sec}$ (100 frames) for single molecule assay. The images were then analyzed by Lab view 8.5 from National instruments using the Kymograph function. A line is drawn along the length of the intended microtubule to be analyzed to get a kymograph. A line is drawn over the events in a kymograph to get the no of pixels that it has travelled. In this case 1 pixel corresponds to $160 \mathrm{~nm}$. The pixels were converted into $\mathrm{nm}$ by multiplying the number of pixels with $160 \mathrm{~nm}$. Velocity was 
then obtained by dividing the total displacement by time travelled in sec. Pauses were calculated in seconds by drawing a line over the stationary particle.

\subsection{ATPase assay}

ATPase measurements were carried out using modified protocol of Huang and Hackney‘s standard protocol (Huang \& Hackney, 1994). The measurements were determined at $25^{\circ} \mathrm{C}$ using the change in absorbance at $340 \mathrm{~nm}$ in a reaction mixture consisting of P12 buffer supplemented with the components in the following order.

$\begin{array}{ll}\text { Assay components } & \text { Volume } \\ \text { 100mM ATP } & 1 \mu \mathrm{l} \\ \text { NADH/PEP } & 4 \mu l \\ \text { PK/LDH/KCl } & 2 \mu l \\ \text { MT } & \text { x } \mu l \\ \text { 1X P12 Buffer } & \text { add 99-x } \mu 1 \\ \text { Motor } 1 \mathrm{mg} / \mathrm{ml} & 1 \mathrm{ul}\end{array}$

The reactions were carried out with varied concentrations of microtubules $(0.5 \mu \mathrm{M}$ $12.0 \mu \mathrm{M}) .1 \mathrm{mg} / \mathrm{ml}$ concentrations of all the UNC-104 and SYD-2 constructs were used in all the experiments. For the experiments with SYD-2 1:1 and 2:1 concentration of motor and SYD-2 were used respectively. The motor and SYD-2 were incubated on ice prior to the addition of the assay components. The OD measurements were taken for 
300s and were plotted as a Time- Course graph with time on the X-axis and OD on the Y-axis. No significant change in OD was seen for either microtubules alone or the motor alone which were used as controls. The change in OD for 60 s was converted to $\operatorname{Kcat}\left(\mathrm{s}^{-1}\right)$ values and plotted against microtubule concentration in $\mu \mathrm{M}$.

\subsection{Blue Native Poly Acryl amide Gel Electrophoresis}

\section{(PAGE)}

\subsubsection{Principle of BN PAGE}

The blue native electrophoresis protocol was devised by Schägger and von Jagow in order to analyse the respiratory chain complexes of mitochondria (Schägger and Von Jagow, 1999). It employs the use of Coomassie dye as a substitute for bound detergent. There by the protein complexes become charged (the dye is negative) and are not present anymore in a detergent micelle, but still remain soluble. The advantage is that one can estimate molecular weight of the membrane protein complex (which is normally not possible due to the bound detergent). This technique also works for soluble proteins in most cases. 


\subsubsection{Preparation of gel}

A 6-16.5\% polyacrylamide gradient gel was prepared a day before as mentioned below.

\begin{tabular}{llll}
\hline$\%$ & 6 & 16.5 & Stack \\
\hline 3xgel buffer & 3 & 3 & 2.5 \\
Acrylamide & 1.07 & 3.05 & 0.6 \\
Glycerol & - & 1.8 & - \\
$10 \%$ APS $(\mu \mathrm{l})$ & 38 & 30 & 30 \\
TEMED $(\mu \mathrm{l})$ & 3.8 & 3 & 3 \\
Water & 4.888 & 1.117 & 4.367 \\
& & & \\
\hline 3X Gel buffer: $(200 \mathrm{mM}$ e-Amino n-caproic acid, 150mM Bis-Tris/HCL pH 7.0)
\end{tabular}

A simple gradient maker with pump is used and a Hoeffer gel system (SE 600 Series, Pharmacia) is employed to cast a gel.

\subsubsection{Preparation of the sample}

The samples were prepared as they were for the pull downs. $10 \mu 1$ of UNC-104 constructs and SYD-2 were incubated on ice for 1 hour before applying to BN PAGE. 3 $\mu l$ loading dye 10x (5\%(w/v) Coomassie Brilliant Blue G-250, 100 mM Bis-Tris pH 7.0, $500 \mathrm{mM}$ 6-aminocaproic acid) is added to $45 \mu \mathrm{l}$ sample (supernatant) and the samples 
are directly applied to Blue Native-PAGE using a syringe. Marker proteins used are thyroglobulin (669 kDa), apoferritin (443 kDa), catalase (230 kDa), alpha-amylase (200 kDa), BSA (66 / 132 kDa) and gamma globulin (39 kDa) (10 $\mu$ g each).

\subsubsection{Electrophoresis}

Cathode buffer (containing 15 mM Bis-Tris pH7.0, $50 \mathrm{mM}$ tricine, 0.02\% Coomassie Brilliant Blue G250) was gently overlaid on top of the samples after they are loaded and electrophoresis is as follows:

A $6-16.5 \%$ polyacrylamide gradient-gel (with $4 \%$ stacking-gel) in $50 \mathrm{mM}$ Bis-Tris pH7.0, $66 \mathrm{mM}$ 6-aminocaproic acid is run in a cooled $\left(4^{\circ} \mathrm{C}\right)$ gel-chamber (Hoefer, SE600) at $100 \mathrm{~V}$ through stacking gel (stay below $15 \mathrm{~mA}$ ) and afterwards at $600 \mathrm{~V}$ through gradient gel (total electrophoresis time $=$ about 5 hours). The gel temperature is maintained at 4 degrees using a cold water pump and magnetic stirrer. After the blue dye has electrophoresed about half way through the gel, the cathode buffer was replaced with fresh cathode buffer containing no coomassie dye (this enables the proteins to become visible in the gel and prevents excess coomassie from interfering with western transfers and subsequent detection). The electrophoresis run was stopped when the front is about $1 \mathrm{~cm}$ above the bottom. 
The gels were subsequently used for western transfer and blotting. The transfer and western protocol are the same as mentioned previously except for the transfer that was done at 220mA for 90’. 


\section{Results}

\subsection{Truncated UNC-104 and SYD-2 proteins interact in vitro.}

The in vitro interaction of the mouse homologue KIF1A and Liprin- $\alpha$ has been reported by Shin et al. According to their report a 455-1104 amino-acid construct of Liprin- $\alpha$ binds best to UNC-104 homologue KIF1A stalk domain at amino acid position 6571105 (Liprin- $\alpha$ binding domain) (Shin et al, 2003). The maximum binding efficiency between similar constructs has been previously reported by our group in C. elegans in a yeast 2 hybrid screen (Wagner et al, 2009). Though UNC-104 and SYD-2 were able to weakly interact through various domains, C-terminal half of SYD-2 was shown to interact prominently with the stalk and FHA domain of UNC-104 (Figure 9A; Wagner et al, 2009). The inramolecular interaction between the stalk and FHA domain of the mouse homologue KIF1A was shown to negatively regulate the motor activity (Lee et al, 2004). The same self regulatory mechanism was also reported for the conventional kinesin/ Kinesin1 by two different groups (coy et al, 1999; Freidman \& vale, 1999) and 
the binding to the cargo or interacting protein was suggested to release the motor from inhibition. Since SYD-2 has been proven to interact with these domains that are involved in self inhibition, we propose that it is a potential candidate to regulate UNC104 activity.

UNC-104 constructs without the FHA domain UNC-104446-GFP-His6 (U446), with the FHA domain UNC-104 ${ }_{653}$-GFP-His6 (U653) and with FHA + stalk UNC-104 ${ }_{800}$-GFPHis6 (U800) were selected to evaluate the regulatory role of SYD-2 (aa608-1089) (Figure 9B). We performed pull down assays with the recombinantly purified SYD-2 and the UNC-104 fragments to check the binding efficiency in vitro. We see significant binding between all the UNC-104 proteins and SYD-2 (aa608-1089) confirming the previous data from yeast 2 hybrids. Incubations of the UNC-104 constructs with either bead alone or with MBP bound to amylose beads as controls (blots not shown). While the longer fragments U800 and U653 were pulled down efficiently, U446 was found to be less efficiently pulled down by MBP-SYD-2 (aa608-1089) (Figure 10). 
A

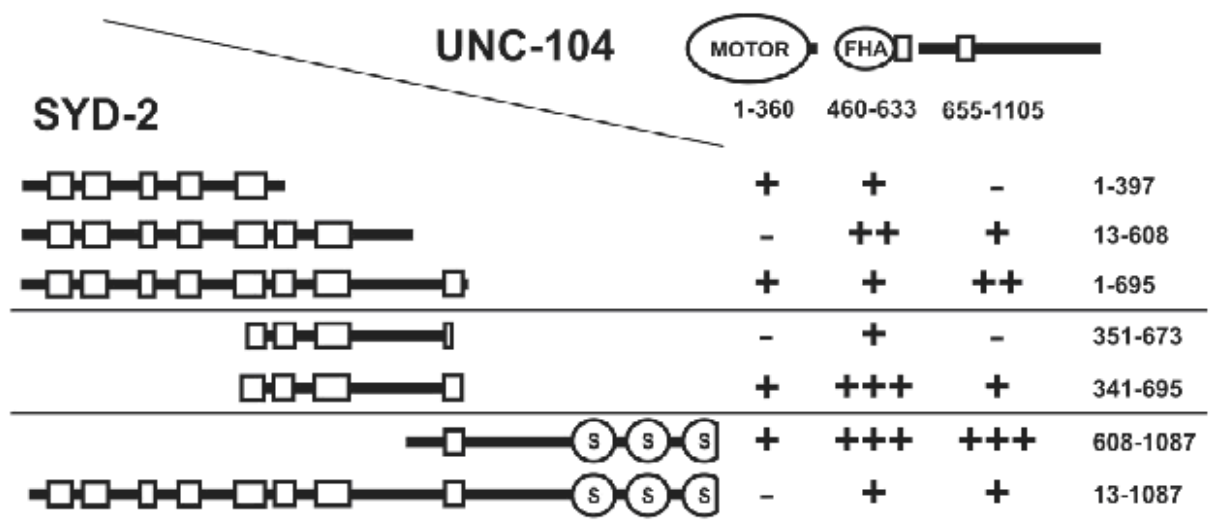

Figure 9A. Yeast 2 Hybrid results from Wagner et al, 2009. The SYD-2 fragment aa608-1087 showed maximum binding to the UNC-104 FHA domain and the stalk region. 

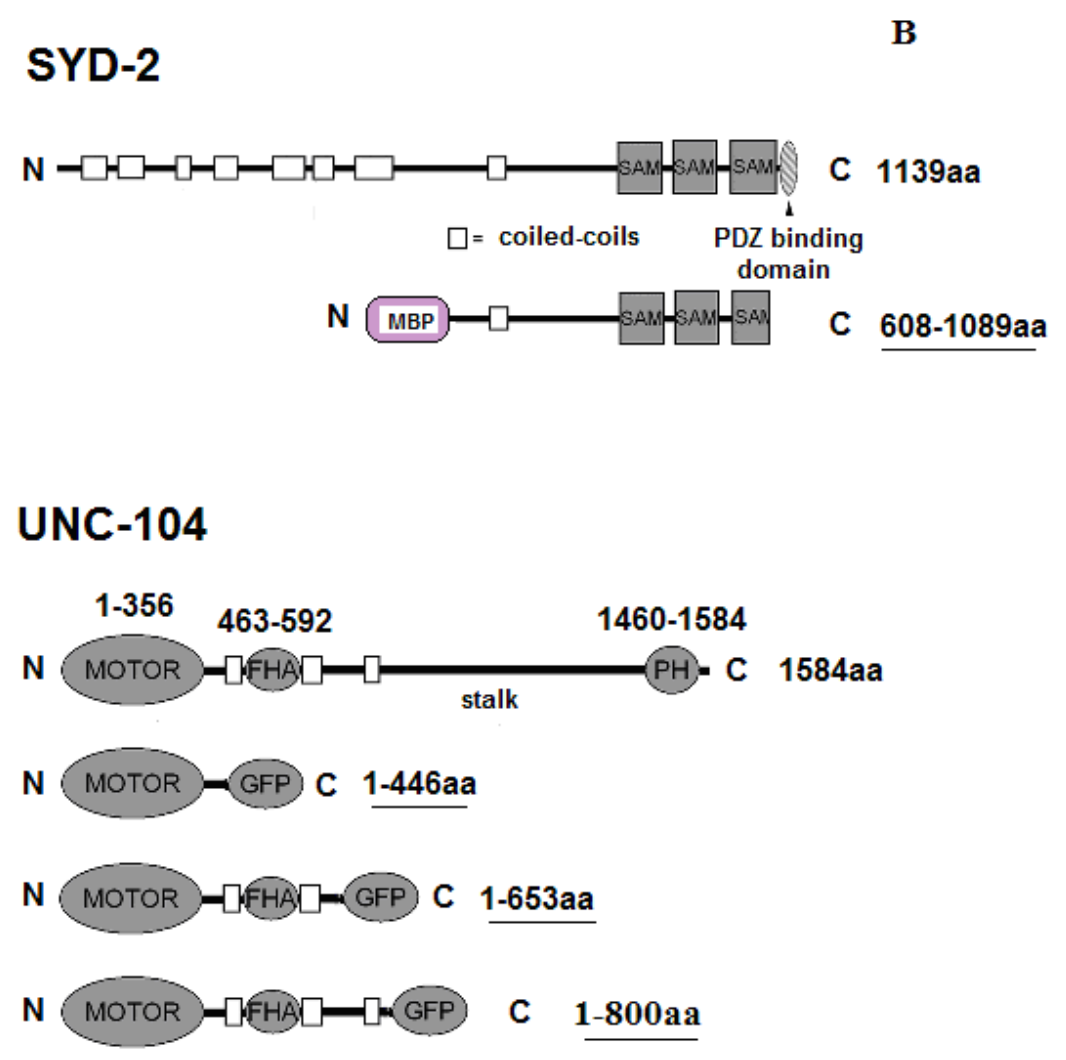

Figure 9B. Schematic representation of the SYD-2 \& UNC-104 domains used in the study (underlined) and the full length structures. 


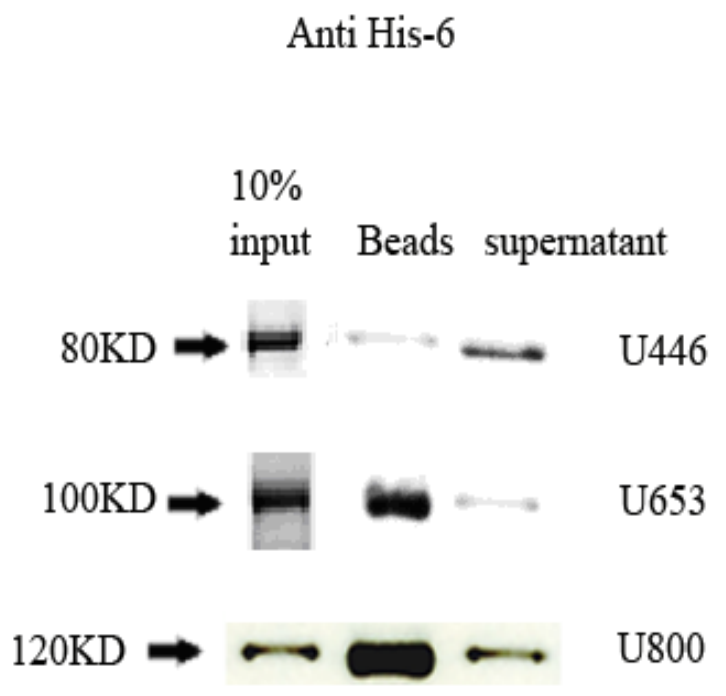

Anti SYD-2 C-terminus

$10 \%$

input Beads supernatant

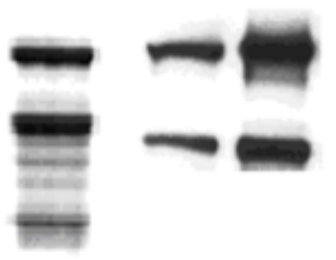

Figure 10. Pull downs of UNC constructs: UNC-His6 blots (above) \& MBP-SYD-2 608-1089 (below). U446 was pulled down less efficiently with SYD-2 in comparison to the other 2 longer constructs. (5\% input in case of U800). 


\subsection{FHA domain is essential for faster UNC-104 movement.}

Having confirmed the in vitro interaction of the UNC fragments with the truncated SYD-2 (aa608-1089), we performed the microtubule gliding assay to characterize the motor activity of the truncated UNC-104 constructs. All the experiments were carried out at $5 \mathrm{mg} / \mathrm{ml}$ motor concentrations. The U653 construct (+FHA domain) translocated the microtubules with $\sim 2$ fold higher velocity than the shorter fragment U446 $₫$ FHA) (Figure 11). The U653 motor moved with a velocity of approximately $1.5 \pm 0.13 \mu \mathrm{m} / \mathrm{sec}$, whereas the U446 moved with a velocity of $0.8 \pm 0.16 \mu \mathrm{m} / \mathrm{sec}$. The average velocity of the longer construct U653 is in the range reported for UNC-104 full length in vivo and in

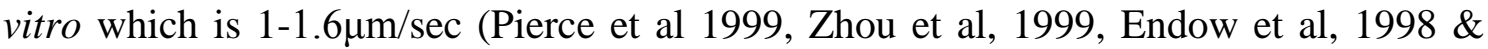
Wagner et al, 2009). The U653 construct had been used in various domain functional studies and our detected velocity is in accordance with the velocities reported in other studies (pierce et al, 1991; Al Bassam et al, 2003). However the velocity of U446 reported by Al Bassam et al (2003) was $2 \mu \mathrm{m} / \mathrm{sec}$ and $2.4 \mu \mathrm{m} / \mathrm{sec}$ for U653. The velocity of truncated KIF1A (aa1-491) protein was reported to be $0.81 \mu \mathrm{m} / \mathrm{sec}$ by Hammond et al (2009). U800 construct which was used to see if the extra stalk changes the motor activity alone moved with a velocity of $3.7 \pm 0.3 \mu \mathrm{m} / \mathrm{sec}$ twice faster than U653(Figure 12 and Table 4).

The FHA domain has been proposed to play a role in stabilizing the dimers formed via the neck region in C. elegans (Al Bassam et al, 2003) and in self inhibition as a result of 
its interaction with CC domain in the stalk for the mouse homologue KIF1A (Lee et al, 2004). The absence of a long stretch of stalk region in both the fragments leaves the dimerization to consider. Though it's hard to determine the exact mechanism or reason for this decrease in velocity, one could only infer that the decrease in U446 velocity might be due to the failure to form stable dimers. The functional importance of the interaction between SYD-2 and UNC-104 has been extensively studied in various model organisms (Wagner et al, 2009). Both of them are dependent on each other to fulfill their specific roles in the neurons. SYD-2 was proposed to act as a linker to the other synaptic proteins (ELKS, GIT1, CASK, Veli and MINT) it interacts with. While this proposal is still accepted, we wanted to see if SYD-2 has any regulatory effect over the motility properties of UNC-104 in vitro. 

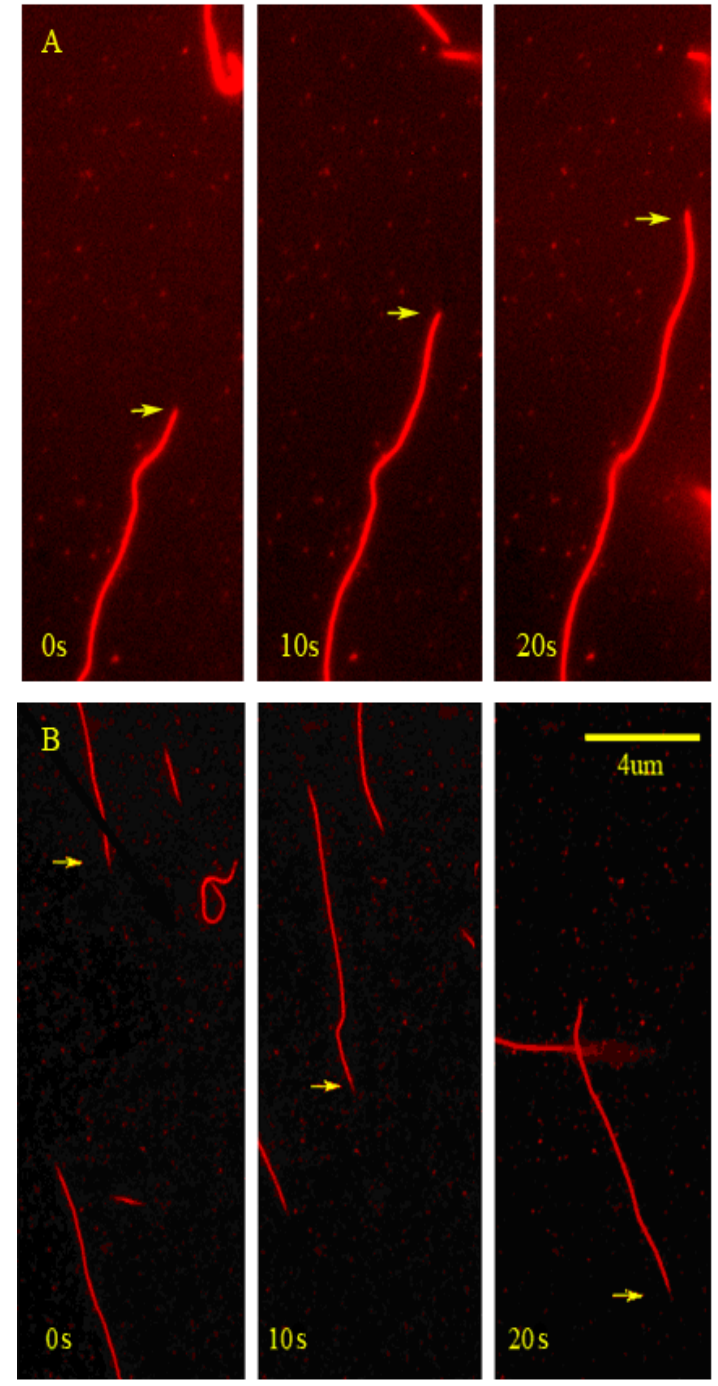

Figure 11. Gliding assay of UNC-104 construct U653-GFP-His6. Time lapse images of U653 alone (A) and after addition of SYD-2 (B). The figures show the movement of rhodamine labeled microtubules over the motor at different time intervals. The arrows represent the displacement of the microtubule end. Images for other constructs not shown. 


\subsection{SYD-2 positively regulates UNC-104 activity}

To elucidate the regulatory effect of SYD-2 we performed the microtubule gliding assays in the presence and absence of truncated SYD-2 (608-1089). All the experiments with individual UNC constructs in the presence and absence of SYD-2 were performed on the same day under same conditions to avoid any discrepancies in the experimental procedures. All the experiments involving SYD-2 were carried out with prior incubation of SYD-2 and UNC proteins on ice for 20 min at 1:1 ratio concentration. The velocities when using motor alone remained the same. Incubation of the U446 motor with SYD-2 did not show any variation in the motor velocity whereas U653 showed a 2 fold increase in velocity from $1.5 \mu \mathrm{m} / \mathrm{sec}$ to $3 \mu \mathrm{m} / \mathrm{sec}$ and U800 showed $30 \%$ increase in velocity upon incubation with SYD-2 (Figure 12). These results point towards a probable regulatory role of SYD-2 involving the FHA domain of UNC-104. The experiments were repeated with 3 different protein preparations of both UNC-104 and SYD-2 and the results were consistent for all the experiments except for very minor insignificant variations in velocity.

SYD-2 did not affect the velocity of the shorter motor fragment U446 probably because of the low binding efficiency between the two as was seen in the pull downs. Another explanation could be the availability of a very short stretch of the neck for interacting 
with SYD-2. The microtubule gliding assays involve the attachment of the motors to the cover slip via the tail domain with the motor heads free to interact with the microtubules. With U653 there is a substantial region ( 300aa) available for interaction with SYD-2 after adsorption to the cover slip, while there is only a short region (<100aa) available for binding to SYD-2 after adsorption in case of U446. This short stretch might restrict the effect of SYD-2 on the U446 construct.

The high velocities in the microtubule gliding assay does not mean that a motor is processive. In the microtubule gliding assays the resulting velocities are the cumulative effect of many motors working in unison. Since the microtubule gliding assay involves use of concentration high enough to saturate the microtubules so that they glide smoothly over the motor lawn we decided to test the constructs in a single molecule assay using total internal reflection microscopy where the motility properties of single molecule could be assessed. Moreover previous work by different groups on either KIF1A or UNC-104 reported high motility velocities in the in vitro microtubule gliding assays or in vivo but none of them resulted in processive movements in the single molecule assays by TIRF. Conventional kinesin that exhibits lower velocities than UNC-104 has shown high processivity in these assays. 

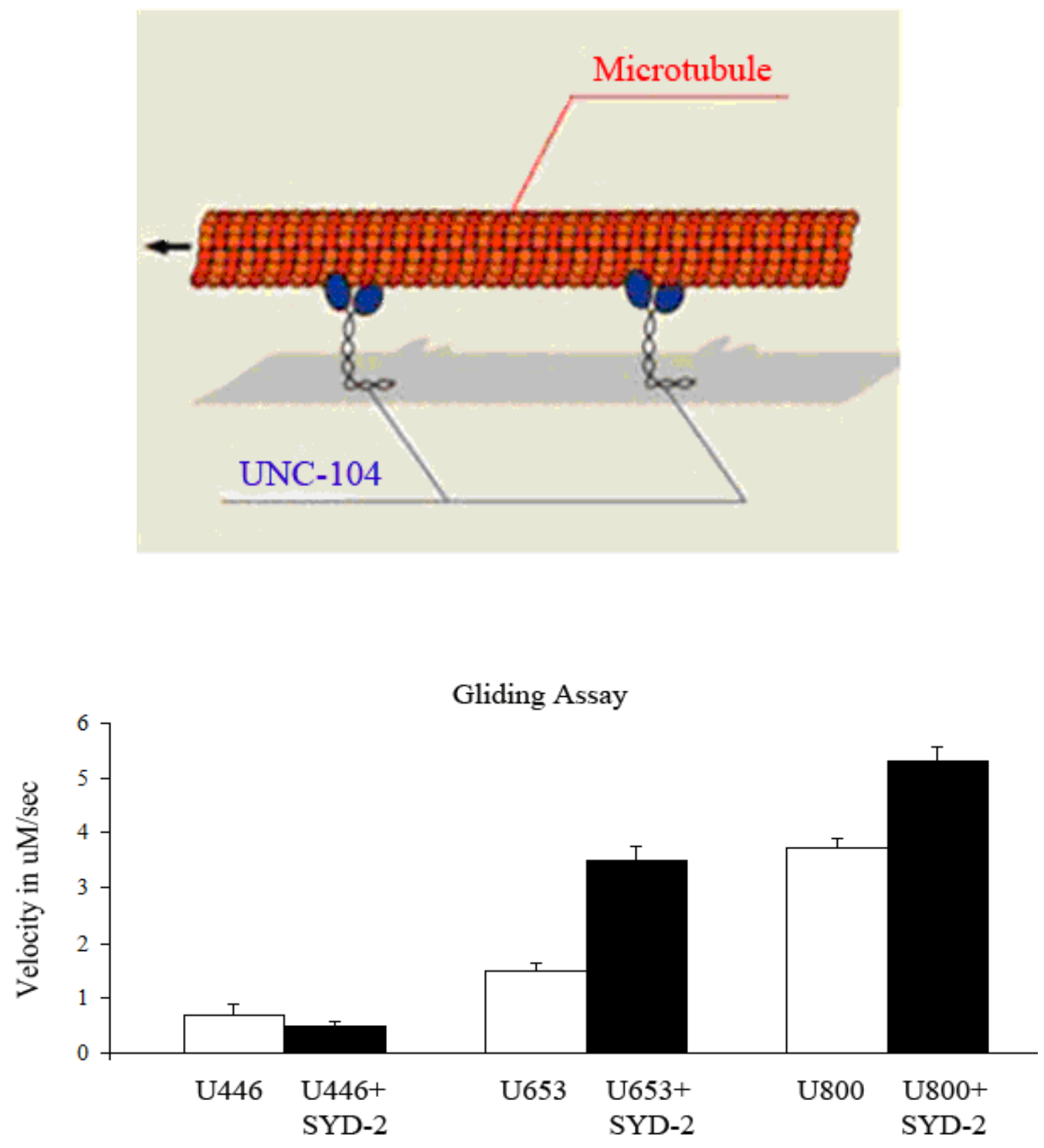

Figure 12. Effect of SYD-2 on the velocity of UNC proteins in gliding assay: Above: Diagrammatic representation of the gliding assay. Below: Graphical representation showing the effect of SYD-2 on the velocity of UNC proteins U653 and U800. Both the UNC proteins showed a significant increase in the presence of SYD-2. The U446 construct did not show increase in velocity in the presence of SYD-2. 


\begin{tabular}{cccc}
\hline Motor & Velocity in $\mu \mathbf{m} / \mathbf{s e c}$ & STD & MTs analyzed \\
\hline U446 & 0.7 & 0.2 & 62 \\
U446+SYD-2 & 0.5 & 0.08 & 78 \\
U653 & 1.5 & 0.13 & 54 \\
U653+SYD-2 & 3.5 & 0.25 & 65 \\
U800 & 3.72 & 0.3 & 96 \\
\hline U800+SYD-2 & 5.3 & 0.28 & 95 \\
\hline
\end{tabular}

Table 4. Motor velocities in gliding assays: Addition of SYD-2 showed 50\% increase in velocity for U653 and 30\% in the case of U800. U446 showed a 40\% decrease in velocity which we assume is due to short neck region available for the head displacement upon SYD-2 binding. (STD: Standard Deviation \& MT: Microtubules) 


\subsection{SYD-2 regulates UNC-104 activity in a single molecule assay by TIRF microscopy}

The motility properties of single motor molecules were tested using TIRF microscopy which is a well established technique to visualize single molecules and analyze their motility behavior. To observe the single motor motility the GFP tagged motor proteins were diluted to nanomolar concentration in a buffer containing $1 \mathrm{mM}$ ATP and flown into the chamber with rhodamine labeled microtubules immobilize onto DETA coated cover slips. By using TIRF microscopy moving spots corresponding to individual motor molecules were visualized and images captured over a 10sec time period (1frame/0.1 sec acquisition). The shorter motor U446 molecules were seen bound to the microtubules and scattered around without any motility which has already been reported for similar constructs (Pierce et al, 1999, Okada et al, 1999). Surprisingly the longer construct U653 exhibited processive unidirectional movement along the microtubules. The same construct was not shown to be processive by Pierce et al (1999) but was processive in our case. Analysis of the motor velocity revealed higher velocities than that were obtained in the microtubule gliding assays. The mean velocity for the U653 motor was $2.79 \pm 0.6 \mu \mathrm{m} / \mathrm{sec}$ and for U800 was $3.9 \pm 0.43 \mu \mathrm{m} / \mathrm{sec}$ (Figure 13 \& Table 5).

In order to check whether the association of SYD-2 has any effect on processivity we repeated the single molecule assays now including SYD-2. We followed the same 
experimental procedures as in the microtubule gliding assay. Here too we performed a prior incubation of SYD-2 and UNC motors on ice at 1:1 concentration. In the assays with the shorter fragment U446, SYD-2 had no effect on the processivity. No events were observed with U446 even in the presence of SYD-2. However the U446 molecules were bound to the microtubules and the cover slip. On the other hand the association of SYD-2 with U653 increased the velocity by $\sim 35 \%(3.8 \mu \mathrm{m} / \mathrm{sec})$ fold comparable to the gliding assay results. Since the incubation of SYD-2 did not have any effect on the construct with the deleted FHA domain but enhanced the velocity of the FHA construct by 2 fold we confirm that SYD positively regulates the UNC-104 motor activity.

One plausible explanation could be conformational changes in the FHA domain that somehow has effects and enhances the motor activity. Since U653 construct has been predicted to be dimer (Al Bassam et al, 2003) the SYD-2 might be involved in stabilizing these dimers. In order to see if this increase in velocity is somehow associated with increased catalysis rate of the motor we assayed the ATPase activity of both the motors once again alone and after incubation with SYD-2. 


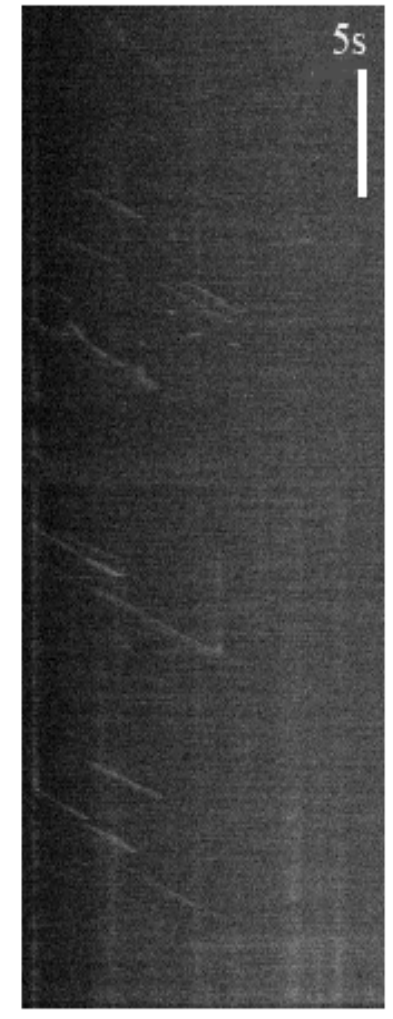

U653

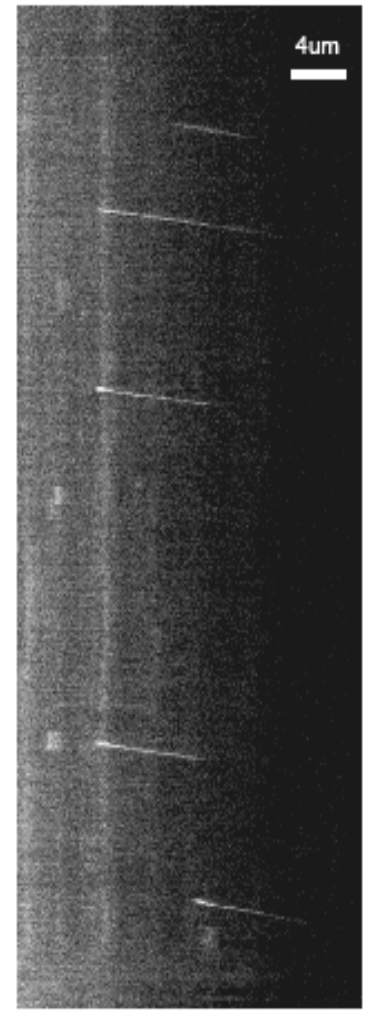

U653+SYD-2

Figure 13. Representative kymographs of U653 in a single molecule analysis. To the left U653 alone and right: after incubation with SYD-2. The image sequence was acquired at $0.1 \mathrm{~s} /$ frame for $100 \mathrm{~s}$. 

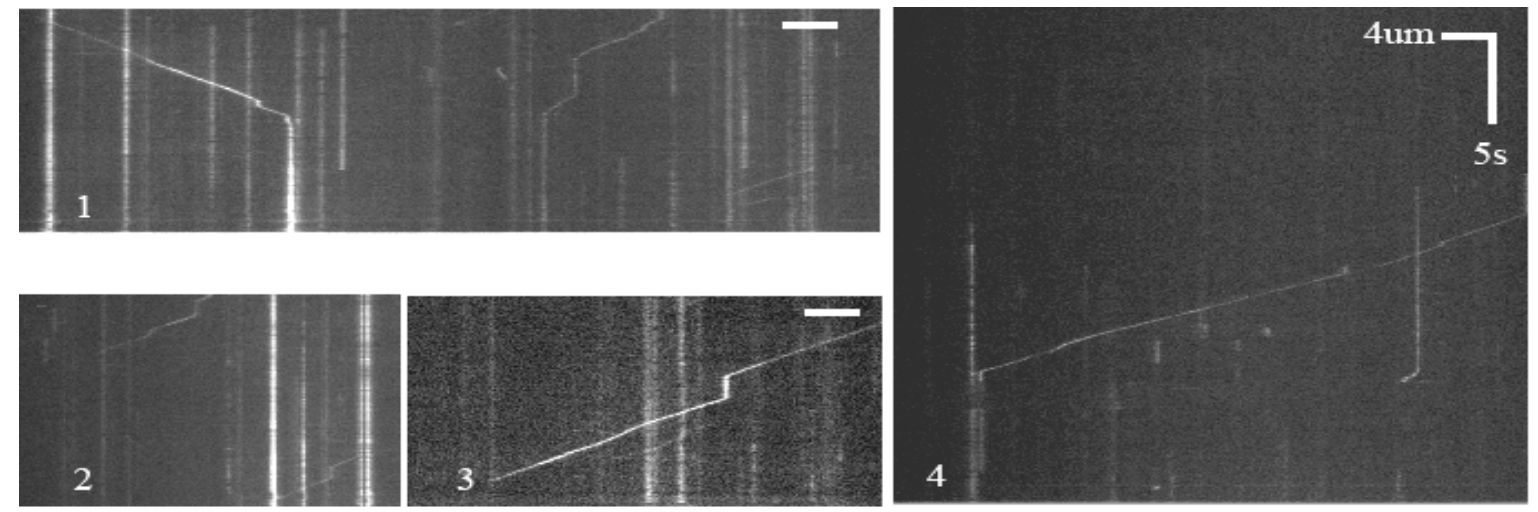

Figure 14. Representative kymographs of U800 (1-2), U800 in the presence of SYD-2 (3-4). The images were acquired at 0.2 s/frame for 100 s. Static particles can be seen as vertical lines whereas the slope of moving particles corresponds to the velocity of the particle 


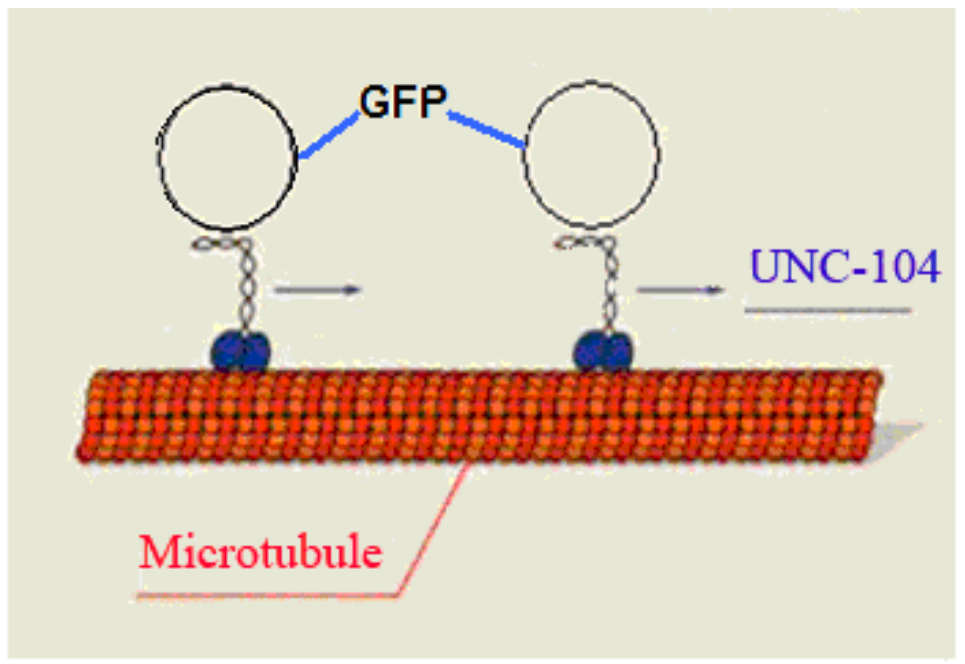

Single molecule assay by TIRF

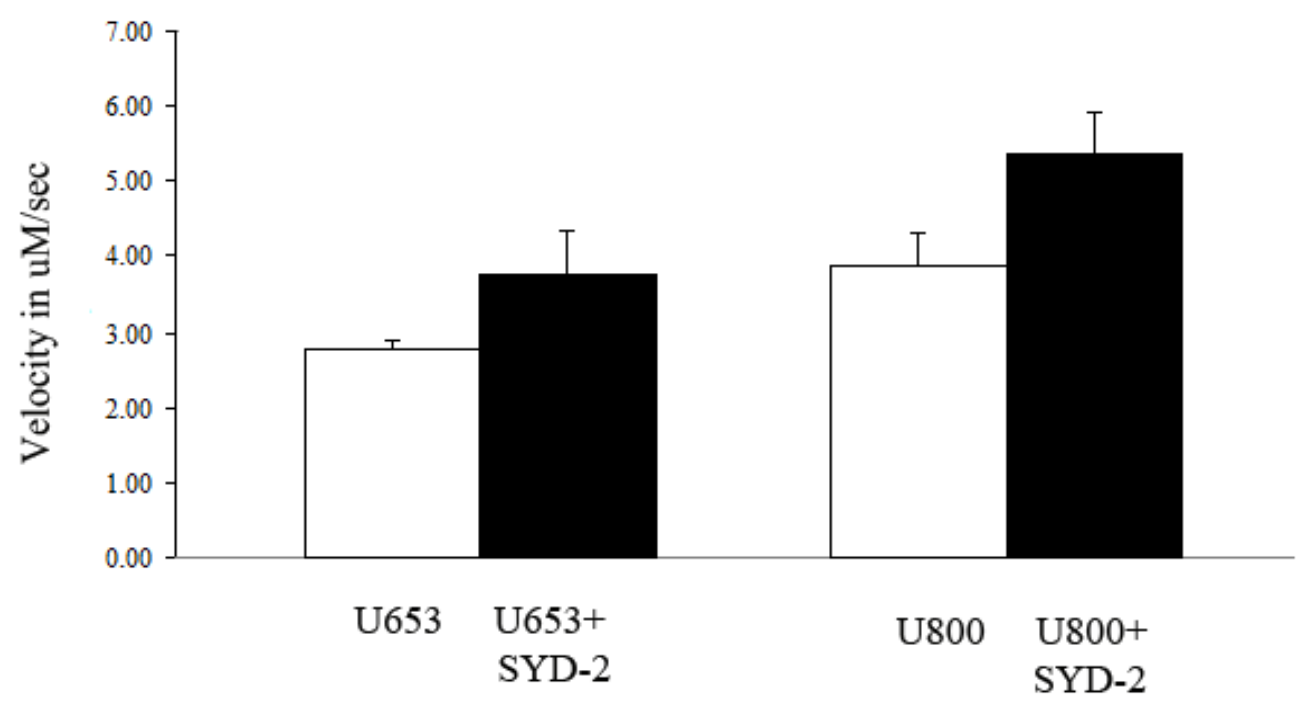

Figure 15. Effect of SYD-2 on the velocity of UNC proteins in single molecule assay: Above: Diagrammatic representation of the Single molecule assay. Below: Graphical representation showing the effect of SYD-2 on the velocity of UNC proteins U653 and U800. U446 did not show any motility 


\begin{tabular}{cccccc}
\hline UNC & Processive & Run Length in $\boldsymbol{\mu m}$ & Velocity in $\boldsymbol{\mu \mathbf { m }} / \mathbf{s e c}$ & Pauses & events analyzed \\
\hline U446 & No & No & - & - & - \\
U446+SYD-2 & No & No & - & - & - \\
U653 & Yes & $2.44 \pm 0.62$ & $2.79 \pm 0.6$ & No & 29 \\
U653 + SYD-2 & Yes & $3.62 \pm 0.55$ & $3.78 \pm 0.54$ & No & 35 \\
U800 & Yes & $1.02 \pm 0.43$ & $3.9 \pm 0.43$ & Yes & 40 \\
U800 + SYD-2 & Yes & $2.6 \pm 0.55$ & $5.37 \pm 0.55$ & Yes & 31 \\
\hline
\end{tabular}

Table 5. Motility properties of the UNC constructs in single molecule assay. No motility events were seen in the experiments with U446 before and after addition of SYD-2. U653 \& U800 showed processivity even in the absence of SYD-2. Addition of SYD-2 showed an increase in velocity by $35 \%$ in U653 and by $27 \%$ in U800. 


\subsection{SYD-2 decreases the catalysis rate of UNC-104}

Steady state ATPase assays were performed to check if the increase in motility rates was due to an increase in ATPase activity. We incubated 1:1 molar concentration of motor prior to addition to microtubules for the experiments including microtubules. No prominent catalysis was seen for either motor alone or microtubules. In the absence of SYD-2 the U446 ( $\triangle \mathrm{FHA}$ ) showed catalysis rate of $~ 5$ ATP per head. While the U653 alone catalyze ATP at the rate of 3.45ATP per head and U800 catalyzes 3.08 ATP per head (Table 6). Previous report by Pierce et al (1999) on the U653 showed that it catalyzed ATP hydrolysis at 5.5 ATP per head. In the presence of ATP we expected to see an increase in the catalysis rate but all the constructs showed a decrease in ATP hydrolysis and an increased $\mathrm{K}_{\mathrm{m}}$. These results somehow do not correlate to the velocities that we measured in the presence of SYD-2. Moreover SYD-2 seems to competitively bind to microtubules. However decrease in SYD-2 concentration as can be seen for U800 showed a decrease in microtubule affinity and a slight increase in ATP concentration.

At this point we are not able to make a solid statement but we believe that the concentration of SYD along with the binding to the motor FHA domain regulates UNC104 velocity and catalysis rate. 


\begin{tabular}{ccc}
\hline Motor & $\mathbf{K}_{\mathbf{m}} \mathbf{M T}(\boldsymbol{\mu M})$ & Kcat $\left(\mathbf{S}^{-\mathbf{1}}\right)$ \\
\hline U446 & $0.38 \pm 0.1$ & $5.48 \pm 0.34$ \\
U446+SYD-2 & $0.72 \pm 0.2$ & $3.61 \pm 0.3$ \\
U653 & $2.2 \pm 0.8$ & $3.45 \pm 0.49$ \\
U653+SYD-2 & $2.36 \pm 0.97$ & $2.94 \pm 0.47$ \\
U800 & $4.3 \pm 1.89$ & $3.1 \pm 0.38$ \\
U800+SYD-2 & $5.35 \pm 2.32$ & $3.08 \pm 0.7$ \\
\hline U800+0.05 $\mu$ MSYD-2 & $4.34 \pm 1.86$ & $3.81 \pm 0.85$ \\
\hline
\end{tabular}

Table 6. Enzymatic characterization of UNC-104 constructs. The ATPase assay of UNC-104 constructs in the presence and absence of SYD-2. $0.1 \mu \mathrm{M}$ Motor was used in the assay. 
Further structural studies involving motor and SYD-2 might shed light as to the conformational changes in the motor domain that results in decreased ATP catalysis and increased motility. One explanation could be the formation of dimers where in you have two motors bound to 2 SYD-2 molecules resulting in a tetrameric structure. Since SYD2 needs the SAM domains to bind to the motor and the $\mathrm{N}$-terminus coiled-coil for binding to itself it might show low affinity to microtubules and thus higher velocities. But this model is still a hypothesis that needs to be tested.

\subsection{Increase in velocity is not due to formation of UNC-104}

\section{dimers}

Since the ATPase assay results were negative we resorted to BN PAGE to see if UNC motors form dimers in the presence of ATP. UNC-104 is a monomer in vivo and though has been proposed to form dimers over lipid membranes or at high concentration; no solid evidence exists till date. Since interaction with SYD-2 increases the processivity and velocity of the motors we doubted if this was due to multimerizing property of SYD-2 resulting in UNC-104 clusters. To test this we preformed blue native

polyacrylamide gel electrophoresis (BN PAGE) to see if U653 shifts to a higher 
molecular weight along with SYD-2 (608-1089). BN PAGE employs use of dye coomassie for labeling the proteins and protein complexes under native conditions.

The U653 was incubated with SYD-2 for 60min on ice. The incubated sample was then subjected to BN PAGE. SYD-2 and U653 were used as controls. U653 was not seen to mobilize to a higher molecular weight in the presence of SYD-2. Though 2 bands were seen around 600KD (Figure 16) in the lane loaded with U653 and SYD-2 incubated sample we were not able to detect it with His6 antibodies in the western blot. We repeated the experiment with high concentration of all the 3 UNC constructs after incubation with SYD-2 but no signal was detected other than the normal size. Thus we conclude that the increase in processivity is not due to dimerization of UNC-104 upon binding to SYD-2. One explanation could be that the dimer formation needs the motor interaction with microtubules 


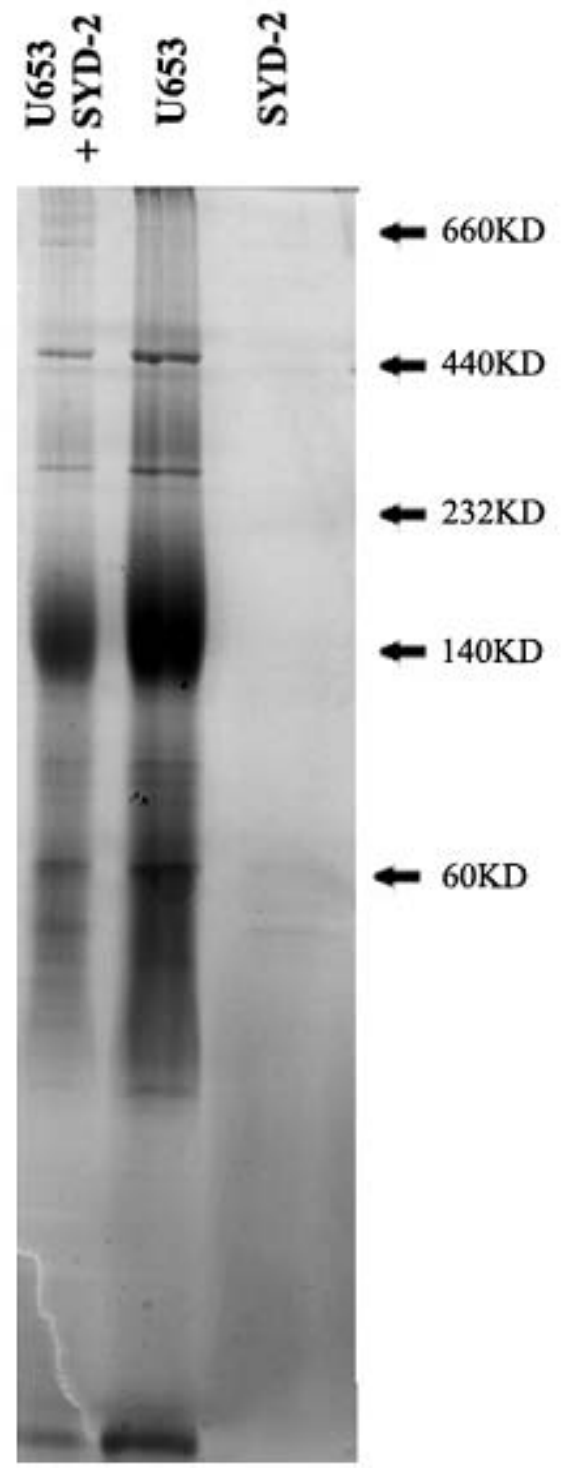

Figure 16. BN PAGE gel image stained with Coomassie blue. The original size of U653 can be seen at around $120 \mathrm{KD}$ and 2 prominent bands can be seen in the left lane at around 600KD. 


\section{Discussion}

UNC-104 is a C. elegans ortholog of mouse kinesin KIF1A and is involved in the anterograde transport of synaptic vesicles along microtubules in the axons in an ATP dependant manner. UNC-104 is expressed solely in the neurons where it appears to be a processive monomer. Many studies have been focusing on the mechanism behind its processive movement in spite of being a monomer (Tomishige et al, 2002; Lee et al, 2004; Nitta et al, 2004; Hammond et al, 2009). Nonetheless little is known about the mechanism regulating UNC-104. In vivo as well as in vitro studies revealed the self regulation of UNC-104 through its FHA domain. It was shown to auto inhibit its activity by binding to its own CC2 domain (Lee et al, 2004). In this work we focus on studying the interaction between UNC-104 and a potential regulatory molecule SYD-2, an active zone protein involved in synaptogenesis. Previous work from our group used C. elegans as a model to understand the importance of their interaction (Wagner et al, 2009) and here in this study we focus on the in vitro assays to decipher the mechanism of the effect of SYD-2 on C. elegans motor protein UNC-104. 


\subsection{UNC-104 interacts with SYD-2 in vitro}

Our previous data from the Yeast 2 Hybrid (Y2H, FIG9A, Wagner et al, 2009) have shown that the FHA (Fork head homology associated) domain containing constructs interacts best with SYD-2 fragment 608-1089 amino acids. FHA domain of KIF1A a mouse homolog of C. elegans UNC-104 has been previously shown to auto-inhibit motor activity by binding to the coiled coil domain close to its own neck region. This led us to hypothesize that SYD-2 might have a regulatory effect on UNC-104 by releasing this inhibitory state by binding to FHA domain. To test this hypothesis we selected three UNC-104 constructs (U446, U653 and U800) with varying binding affinity to SYD-2 (608-1089) to analyze the function of the interaction between these two proteins (FIG9B). The two constructs $\mathrm{U}_{653}$-GFP-His6 (1-653aa) and $\mathrm{U}_{800}$-GFP-His6 (1-800aa) containing the FHA domain showed the highest binding efficiency to the SYD-2 construct 608-1089aa while The $\mathrm{U}_{446}$-GFP-His6 (1-446aa) lacking the FHA domain showed decreased binding efficiency in the Y2H (FIG9A). We performed pull downs of the selected UNC protein with MBP-SYD-2 (608-1089aa) to confirm their interaction in vitro. We saw high binding affinity with the UNC constructs containing the FHA domain. With the U446 lacking the FHA domain we see residual binding though significantly robust in all gliding assays performed (FIG12, Table 4). These 
results confirm our previous data from the $\mathrm{Y} 2 \mathrm{H}$ that the UNC-104 constructs containing the FHA domain exhibit high binding efficiency to SYD-2 C-term fragment (6081089aа).

\subsection{FHA domain is important for faster motility}

The in vivo data from our published work shows that SYD-2 is involved in regulating the UNC-104 motility (Wagner et al, 2009). In syd-2 mutant animals, an increase in the retrograde (-end) movement of synaptic vesicles in the C. elegans axons was observed. It has already been shown that the FHA domain affects the directionality of the motor, by changing the balance of anterograde and retrograde movement in mutants (Wagner et al, 2009). So we hypothesized that SYD-2 might exert its effect by binding to UNC-104 FHA domain. To check the affect of SYD-2 on UNC-104 motility we determined the motor velocities of individual UNC proteins in microtubule gliding assays.

The U446 (AFHA) showed decreased velocity than the other 2 constructs with FHA domain. It displaced the microtubules with a velocity of $\sim 0.8 \mu \mathrm{m} / \mathrm{s}$ which is $1 / 2$ the velocity of U653 and $1 / 4$ the velocity of U800. U653 showed an average velocity of $\sim 1.5 \mu \mathrm{m} / \mathrm{s}$ and U800 showed an average $\sim 3.7 \mu \mathrm{m} / \mathrm{s}$ (Table 4 .). The decreased velocity in U446 might be due to the association of the neck region to the glass surface in the microtubule gliding assays. Since the neck region next to the motor domain (1-354aa) is very short and stuck to the glass this might hinder the movement of motor head to some 
extent. It is also possible that the short linker region does not allow for proper orientation of UNC-104 motors on the microtubule surface.

In the case of U800 the extended stalk might be involved in some conformational changes and contribute to increased velocity $(\sim 3.7 \mu \mathrm{m} / \mathrm{s})$ a high velocity value that has

not been reported for UNC-104 whose average velocity yin vitro was 2 um/s (Al Bassam et al, 2004). UNC-104 motors are thought to adopt a monomeric confirmation in vivo but were proposed to form dimers at high concentrations through the coiled-coil domain (Al Bassam et al, 2003). The absence of the stalk after the coiled-coiled domain in U653 might have resulted in the formation of unstable dimers and probably stable dimers in the case of U800. Additional experiments need to test this hypothesis.

\subsection{SYD-2 enhances UNC-104 velocity in FHA containing}

\section{UNC-104 constructs}

To determine the effect of SYD-2 on the truncated UNC-104 proteins we repeated the gliding assays with the UNC-104 constructs in the presence of SYD-2. Incubations with SYD-2 increased the velocity of U653 by 2 fold confirming that SYD-2 does affect the motor velocity positively. The U446 constructF(HA) did not show any change in velocity upon the addition of SYD-2 confirming our hypothesis that the interaction 
between FHA and SYD-2 is involved in motor regulation. Even with the longer fragment U800 we saw a significant increase in velocity after the addition of SYD-2. This suggests that the FHA domain is important for the increase in velocity upon binding to SYD-2.

\subsection{SYD-2 enhances UNC-104 velocity in the single molecule}

\section{assay}

Previous studies showed that the monomeric motor UNC-104 attained high velocity through a week interaction between the K-loop of the motor and C-terminus of tubulin (Okada et al, 2000). This association lead to higher velocities but was not able to walk long distances over a microtubule (not processive). To check whether SYD-2 somehow affects the processivity resulting in higher speed we performed single molecule assays. Single molecule assays offer the possibility to analyze the motor properties in an environment completely different from either in vivo systems or multiple motor assays. While in vivo system describe the function of motors in its physiological context (including all necessary factors), in vitro system like a microtubule gliding assay describe the mechanical properties of multiple motors attached to the microtubule surface. Thus, it is difficult to deduce individual enzymatic properties from multiple motor assays as the processivity (i.e. the number of consecutive movements before 
falling off a microtubule track). However processivity can be measured by single molecule fluorescence microscopy, a method that allows one to distinguish whether motors are only active in an ensemble or as individual entities. Therefore, we looked at the motor movements in the single molecule assay to test if an increase with UNC-104's velocity correlates with higher processivity and thus a more efficient use of ATP. In single molecule assays the U446 ( $\triangle$ FHA) molecule which was not affected by SYD -2 and showed low velocity in the gliding assay (FIG12 \& Table 4.), did not show any processive movement in the single molecule assay using TIRF microscopy. The other two constructs containing the FHA domain exhibited processive motility. Once again the U800 construct showed higher motility rates than the U653. One explanation could be that the stalk region might be involved in some secondary structure formation which might probably influence the catalytic rate of the motor.

One interesting finding was that the velocity of the U653 motor had a significant increase in velocity in the single molecule assay while the U800 velocity was consistent in both the assays. With the addition of SYD-2 we saw $\sim 35 \%$ increase in velocity in both the constructs while U446 did not show any movement along the microtubules.

The presence of SYD-2 increases the motility rate in both U653 and U800 constructs (FIG13-15 \& Table 5). Previous studies with the UNC-104 motor in the single molecule assays showed no processivity (Pierce et al, 1999) except for a recent report from Hammond et al (2009) where they showed that the mouse homolog KIF1A is able to 
move processively in the single molecule assays. We assume that this might be due to the use of higher concentration of UNC-104 in our assays when compared to the previous reports. We also saw considerable increase in run length in both the constructs in the presence of SYD-2 which is important to achieve long range transport in the axons.

Taken together this data suggests that the interaction of SYD-2 with the FHA domain of UNC-104 enhances the motor activity and processivity.

We have ample evidence through in vivo data that the interaction between UNC104/KIF1A with SYD-2/Liprin- $\alpha$ is important for normal functioning of both the proteins (Miller et al, 2005; Shin et al, 2003, Wagner et al, 2009) and no we show that SYD-2 positively regulates UNC-104 activity by increasing its processivity. We have 2 hypotheses for the increased processivity; one is that association of SYD-2 leads to conformational changes in the motor domain leading to increased ATPase activity thus resulting in increased motility rate. The second hypothesis is that the multimerizing property of SYD-2 might cluster 2 UNC-104 molecules together leading to a heterotetramer and thus resulting in increased velocity.

\subsection{SYD-2 decreases the ATPase activity of UNC-104 motor.}

The FHA domain is located close to the active site of the kinesin and this has led to the proposition that the FHA domain might play a role in regulating the catalytic cycle by 
binding to tubulin, the substrate of the kinesin (Kikkawa et al, 2001). Though this hypothesis has not been proven yet we wanted to check if SYD-2 association with UNC104 FHA domain has any effect on catalysis rate which might explain the increase in processivity. We performed the steady state ATPase assay with varying concentrations of microtubules. Here we see that SYD-2 competitively binds to microtubules and thus inhibits the motor. This effect was reduced when the concentration of SYD-2 was reduced.

Probably the effect of SYD-2 is concentration dependant and in biological system there might be other regulators of SYD-2. SYD-2 binding proteins need the N-terminal domains for interaction and then probably the affinity to microtubules is reduced.

We also tried to see if the increase in velocity is due to formation of dimers which might explain the decrease in ATP consumption. To check this we performed BN PAGE with the UNC-104 motors incubated with SYD-2, but we see no higher shift in the molecular weight of UNC-104. Probably this interaction needs binding to microtubules to form stable clusters.

Further studies including UNC-104 and SYD-2 might shed light on the correlation between the competitive inhibition of SYD-2 and increased Velocity. We think that the formation of tetramers is quite possible which are stable in the presence of microtubules and thus the ATP hydrolysis rate is decreased in the presence of SYD-2. Al Bassam et al 
(2003) reported that UNC-104 utilizes high ATP when compared to its displacement in in vitro assays and proposed that the one dimensional diffusion is responsible for this property. In our case probably the clustering of UNC-104 might be moving actively and thus resulting in decreased ATP consumption.

In the future we plan to repeat the single molecule assay with labeled SYD-2 and see if the SYD-2 molecules are transported along with UNC-104 constructs. We also plan to perform cross linking experiments to see if UNC-104 are able to form dimers in vitro in the presence of SYD-2 


\section{Conclusion}

In this work we aimed to understand the mechanism of functional interaction between the kinesin-3 motor UNC-104 and its binding partner SYD-2. Here we show that SYD-2 positively regulates UNC-104 motility in vitro and renders the motor processive. UNC104 FHA domain seems to be critical for the positive regulation. We tried to decipher the mechanism of regulation by using ATPase assay. We hypothesized that increase in ATPase activity might be resulting in increased motor velocities which does not seem to be the case. We see a decrease in ATP hydrolysis rate and increase in binding to microtubules which is hard to explain. Further investigation of the structural and functional aspect of the UNC-104 and SYD-2 might help us to understand the regulatory mechanism. Probably motor domain structural studies involving SYD-2 and microtubules might shed light as for the conformational changes that occur due to binding of SYD-2 in the presence of microtubules. 


\section{References}

Aizawa H, Sekine Y, Takemura R, Zhang Z, Nangaku M and Hirokawa N. (1992) Kinesin family in murine central nervous system. J. Cell Biol 119: 1287-1296.

Al-Bassam J, Cui Y, Klopfenstein D.R, Carragher B.O, Vale R.D, Milligan R.A. (2003) Distinct conformations of the kinesin Unc104 neck regulate a monomer to dimer motor transition. J Cell Biol 163(4): 743-53.

Block S.M, Goldstein L.S, Schnapp B.J. (1990) Bead movement by single kineisn molecules studied with optical tweezers. Nature 348(6299):348-52.

Bloom G.S, Wagner M.C, Pfister K.K, Brady S.T. (1988) Native structure and physical properties of bovine brain kinesin and identification of the ATP-binding subunit polypeptide. Biochemistry 27: 3409-16.

Cai D, Hoppe A.D, Swanson J.A, Verhey K.J. (2007) Kinesin structural organization and conformational changes revealed by FRET stoichometry in live cells. J Cell Biol 176 (1) 51-63.

Coy D.L, Hancock W. O, Wagenbach M. \& Howard J. (1999) Kinesin's tail domain is an inhibitory regulator of the motor domain. Nature Cell Biol 1, 288-292. 
Dai Y, Taru H, Deken S.L, Grill B, Ackley B, Nonet M.L and Jin Y. (2006) SYD-2 Liprin-alpha organizes presynaptic active zone formation through ELKS. Nat Neurosci. 9(12):1479-1487.

Endow S.A and Waligora K.W. (1998) Determinants of kinesin motor polarity. Science 281, 1200-1202.

Friedman D. S and Vale R. D. (1999) Single-molecule analysis of kinesin motility reveals regulation by the cargo-binding tail domain. Nature Cell Biol. 1, 293-297.

Hall D.H. and Hedgecock E.M. (1991) Kinesin-related gene unc-104 is required for axonal transport of synaptic vesicles in C. elegans. Cell 65(5): 837-847.

Hirokawa, N. (1998) Kinesin and dynein superfamily proteins and the mechanism of organelle transport. Science 279, 519-526.

Hirokawa N, Pfister K.K, Yorifuji H, Wagner M.C, Brady S.T, Bloom G.S. (1989) Submolecular domains of bovine brain kinesin identified by electron microscopy and monoclonal antibody decoration. Cell 56: 867-878.

Hirokawa N, Takemura R. (2004) Kinesin superfamily proteins and their various functions and dynamics. Exp Cell Res 301(1): 50-9. Review.

Hirokawa N and Takemura R. (2005) Molecular motors and mechanisms of directional transport in neurons. Nat Rev Neurosci 6: 201-214.

Hollenbeck P.J. (1989) The distribution, abundance and subcellular localization of kinesin. J Cell Biol. 108(6):2335-2342.

Howard J, Hudspeth A. J. and Vale R. D. (1989) Movement of microtubules by single kinesin molecules. Nature 342, 154-158.

Huang T.G and Hackney D.D. (1994) Drosophila kinesin minimal motor domain expressed in Escherichia coli. Purification and kinetic characterization. J. Biol. Chem 269(23): 16493-16501. 
Kamal A, Stokin G.B, Yang Z, Xia C.H and Goldstein L.S. (2000) Axonal transport of amyloid precursor protein is mediated by direct binding to the kinesin light chain subunit of kinesin-I. Neuron 28(2):449-459.

Kamal A, Almenar-Queralt A, LeBlanc J.F, Roberts E.A and Goldstein LS. (2001) Kinesin-mediated axonal transport of a membrane compartment containing betasecretase and presenilin-1 requires APP. Nature 414(6864):643-648.

Kaufman N, DeProto. J, Ranjan. R, Wan. H, and Van Vactor D. (2002) Drosophila Liprin- $\alpha$ and the Receptor Phosphate Dlar control synapse morphology. Neuron 34: 2728

Kikkawa M, Sablin E.P, Okada Y, Yajima H, Fletterick R.J and Hirokawa N. (2001) Switch-based mechanism of kinesin motors. Nature 411: 439-445.

Klopfenstein D.R, Tomishige M, Sturrman N, and Vale R. D. (2002) Role of Phosphatidylinositol $(4,5)$ bisphosphate Organization in Membrane Transport by the Unc104 Kinesin Motor. Cell 109: 347-358.

Klopfenstein D.R and Vale R.D. (2004) The lipid binding pleckstrin homology domain in UNC-104 kinesin is necessary for synaptic vesicle transport in Caenorhabditis elegans. Mol Biol Cell. 2004 15(8):3729-3739.

Ko J, Kim S, Valtschanoff J.G, Shin H, Lee J.R, Sheng M, Premont R.T, Weinberg R.J, Kim E. (2003a) Interaction between liprin-alpha and GIT1 is required for AMPA receptor targeting. J Neurosci 23: 1667-1677.

Ko J, Na M, Kim S, Lee J.R, Kim E (2003b) Interaction of the ERC family of RIMbinding proteins with the liprin-alpha family of multidomain proteins. $J$ Biol Chem 278: 42377-42385.

Lee J.R, Shin H, Choi J, Ko J, Kim S, Lee H.W, Kim K, Rho S.H, Lee J.H, Song H.E, Eom S.H, Kim E. (2004) An intramolecular interaction between the FHA and a coiled coil negatively regulates the kinesin motor KIF1A. EMBO J 23: 1506-1515. 
Lee J.R, Shin H, Ko J, Choi J, Lee H and Kim E. (2002) Characterization of the movement of the Kinesin motor KIF1A in living cultured neurons. J. Biol. Chem. 278: 2624-2629.

Miki H, Setou M, Kaneshiro K and Hirokawa N. (2001) All kinesin superfamily protein, KIF, genes in mouse and human. PNAS 98, 7004 - 7011.

Miller K.E, DeProto J, Kaufmann N, Patel B.N, Duckworth A, Van Vactor D (2005) Direct observation demonstrates that Liprin-alpha is required for trafficking of synaptic vesicles. Curr Biol 15: 684-689.

Nitta R, Kikkawa M, Okada Y and Hirokawa N. (2004) KIF1A alternately uses two loops to bind microtubules. Science 305(5684): 678-683.

Okada Y, Yamazaki H, Sekine-Aizawa Y and Hirokawa N (1995). The neuron specific kinesin superfamily protein KIF1A is a unique monomeric motor for anterograde axonal transport of synaptic vesicle precursors. Cell 81: 769-80.

Okada Y and Hirokawa N. (2000). Mechanism of the single-headed processivity: Diffusional anchoring between the K-loop of kinesin and the $\mathrm{C}$ terminus of tubulin. PNAS 97(2): 640-5.

Olsen O, Moore K.A, Fukata M, Kazuta T, Trinidad J.C, Kauer F.W, Streuli M, Misawa H, Burlingame A.L, Nicoll R.A and Bredt D.S (2005) Neurotransmitter release regulated by a MALS-liprin- $\alpha$ presynaptic complex. J Cell Biol 170: 1127-1134.

Otsuka A.J, Jeyaprakash A, Garcia-Anoveros J, Tang L.Z, Fisk G, Hartshorne T, Franco R, and Born T. (1991). The C. elegans unc-104 gene encodes a putative kinesin heavy chain-like protein. Neuron 6, 113-122.

Patel M.R, Lehrman E.K, Poon V.Y, Crump J.G, Zhen M, Bargmann C.I, and Shen K. (2006) Hierarchical assembly of presynaptic components in defined C. elegans synapses. Nat Neurosci 12:1488-98. 
Pierce D.W, Hom-Booher N, Otsuka A.J, Vale R.D. (1999) Single-molecule behavior of monomeric and heteromeric kinesins. Biochemistry 38(17):5412-5421.

Seog D.H, Lee D.H and Lee S.K. (2004) Molecular Motor Proteins of the Kinesin Superfamily Proteins (KIFs): Structure, Cargo and Disease. J Korean Med Sci. 19: 1-7.

Serra-Pages C, Kedersha N. L, Fazikas L, Medley Q, Debant A, and Streuli M. (1995). The LAR trans-membrane protein tyrosine phosphatase and a coiled-coil LARinteracting protein co-localize at focal adhesions. EMBO J 14: 2827-2838.

Serra-Pages C, Medley Q.G, Tang M, Hart A and Streuli M (1998) Liprins, a family of LAR trans-membrane protein-tyrosine phosphatase-interacting proteins. J Biol Chem 273: $15611-15620$.

Setou M, Seog D.H, Tanaka Y, Kanai Y, Takei Y, Kawagishi M, Hirokawa N. (2002) Glutamate-receptor-interacting protein GRIP1 directly steers kinesin to dendrites. Nature 417(6884): 83-87.

Schägger H and von Jagow G. (1991) Blue Native Electrophoresis for isolation of membrane protein complexes in enzymatically active form. Anal. Biochem. 199: 223231.

Shin H, Wyszynski M, Huh K.H, Valtschanoff J.G, Lee J.R, Ko J, Streuli M, Weinberg R.J, Sheng M and Kim E (2003) Association of the kinesin motor KIF1A with the multimodular protein Liprin alpha. J Biol Chem 278: 11393-11401.

Svoboda K, Schmidt C.F, Schnapp B.Jand Block S.M. (1993) Direct observation of Kinesin stepping by optical trapping interferometry. Nature 365(6448):721-7.

Tomishige M, Klopfenstein D.R, and Vale R.D. (2002). Conversion of Unc104/ KIF1A kinesin into a processive motor after dimerization. Science 297: 2263-2267.

Vale R.D, Reese T.S, Sheetz M.P. (1985) Identification of a novel force generating protein, Kinesin, involved in microtubule-based motility. Cell 42, 39-50. 
Vale R.D. (2003) Kinesin the Molecular Motor toolbox for intracellular transport. Cell 112, 467-480.

Wagner O.I, Esposito A, Koehler B, Chen C.W, Shen C.P, Wu G.H, Butkevich E, Mandalapu S, Wenzel D, Wouters F.S, and Klopfenstein D.R. (2009). Synaptic scaffolding protein SYD-2 clusters and activates Kinesin-3 UNC-104 in C. elegans. PNAS 106: 19605-19610.

Wyszynski M, Kim E, Dunah A.W, Passafaro M, Valtschanoff J.G, Serra-Pages C, Streuli M, Weinberg R.J, Sheng M (2002) Interaction between GRIP and Liprinalpha/SYD-2 is required for AMPA receptor targeting. Neuron 34:39-52.

Yang JT, Laymon RA, Goldstein LS. (1989) A three-domain structure of kinesin heavy chain revealed by DNA sequence and microtubule binding analyses. Cell; 56: 879-89.

Yeh E, Kawano T, Weimer R.M, Bessereau J.L and Zhen M. (2005) Identification of genes involved in synaptogenesis using a fluorescent active zone marker in Caenorhabditis elegans. J Neurosci. 25(15):3833-3841.

Yonekawa Y, Harada A, Okada Y, Funakoshi T, Kanai Y, Takei Y, Terada S, Noda T, and Hirokawa N. (1998). Defect in synaptic vesicle precursor transport and neuronal cell death in KIF1A motor protein-deficient mice. J Cell Biol 141: 431-441.

Zhen M and Jin Y. (1999) The Liprin protein SYD-2 regulates the differentiation of presynaptic termini in C. elegans. Nature 401:371-375. 


\section{Acknowledgement}

I would like to express my gratitude to Dr. Dieter Klopfenstein for his excellent supervision and support throughout my PhD, Without whom this work would not have been possible: to Prof. Ivo Feussner and Prof. Frauke Melchior for being my thesis committee members, for their valuable suggestions and guidance that helped me grow as a researcher, Prof. Christoph Schmidt and his group for their help and support, Human frontiers for their financial support without which this project would not have been possible. I would also like to take this opportunity to convey my heartfelt thanks to my former colleagues, Barbara Koehler, Oliver Wagner and Elke Raade who welcomed me into this wonderful group and made my time in this lab really unforgettable. I will always cherish the moments we spent together. To Eugenia Butkevich, Volker Henschel and Wiebke Anna Sassen for being such good friends and colleagues.

Being far away from India and loved ones all these years would not have been possible if not for the Indian community in Goettingen. I would like to take this opportunity to thank one and all for making my days in Goettingen memorable, especially Sunil and 
Deepa, Rajeshwar Rao, Krishna, Sudhakar, Raghu, Kalyani, Anjana, Vijay and Pooja, adorable Vivin and Nitin, Karthik and Sowmya, Tina, Raunak and many many more.

My sincere thanks to Miriam and Mahshid for being such good friends and making my days in Goettingen quite unforgettable.

To my parents, brother, grand mom and other friends and loved ones back in India for their constant love, support and encouragement.

Last but not least to my beloved husband Sridhar for his undying support love and constant nagging $:$

Thank you one and all for you love and support. 


\title{
Curriculum Vitae
}

\author{
SAILAJA MANDALAPU \\ Drittes Physikalisches Institut \\ Faculty of Physics \\ Friedrich-Hund-Platz 1 \\ 37077 Göttingen \\ Germany \\ Phone - +4917663228508 (mobile)
}

Date and Place of Birth

\section{Education}

\section{B.Sc (Microbiology, Bio-Chemistry, Nutrition)}

Nagarjuna University

Guntur, India

MSc (Bio-Chemistry)

Periyar University

Salem, India

PhD (Bio-Chemistry)

Aug 2006 to present

4 July 1978, Tenali, India

Jun 1995 to Mar 1998

Sep 1998 to May 2000

University of Goettingen

Germany 


\section{Research Experience}

PhD, George August University Goettingen,

Germany

Practical training, Department of

Neuroanotomy, Goettingen, Germany.

Senior Cytogeneticist, Centre for Eugenics, Hyderabad, India.

Junior research fellow, Department of Genetics, Bhagwan Mahavir Hospital and Research

Centre, Hyderabad, India.
Aug 2006 - present

May 2005 - Aug 2005

Nov 2002 - Dec 2003

Oct 2001 - Oct 2002

\section{PhD project}

Regulation of Kinesin-3 activity by active zone protein SYD-2

$\mathrm{PhD}$, Institute for Biochemistry and Physiology, Humboldtalle 23, 37073 Goettingen, Germany. Aug2006 - Present

\section{Practical Training}

Department of Neuroanatomy, University of Goettingen, Kreuzbergring 36, 37075 Goettingen, Germany. May 2005 - Aug 2005

Primary neuronal cell culture, Immunocytochemistry, Immunohistochemistry, tisuue sectioning and processing for Immunohistochemistry, western blotting.

\section{Senior Cytogeneticist}

Department of genetics, Center for Eugenics, Barkatpura, Hyderabad, India. Nov 2002 Dec 2003 
My job Involved setting up the lab, interacting with patients, taking pedigree, processing blood, fetal cord blood, bone marrow, aborted tissue, fetal biopsies and amniotic fluid for chromosomal analysis by karyotyping and counseling patients.

\section{Research project}

\section{“The role of Chromosomal anomalies in repeated abortions".}

The project involved interacting with the couple having repeated abortions, taking detailed pedigree and family history and collecting blood samples for chromosomal analysis (Karyotyping and Genetic mutation screening).

\section{Junior Research fellow}

Department of Genetics, Bhagwan Mahavir Medical Research Center, AC Guards, Hyderabad. Oct 2001 - Oct 2002

During this period I was involved in cytogenetic and molecular diagnostic - this work involved culturing lymphocytes for chromosomal analysis and genomic DNA isolation from blood and tissue samples from patients for PCR and training students in cytogenetics.

\section{Research project}

"In vitro technologies for risk assessment and alternatives to in vivo methods of systemic toxicology" (Council for Scientific and Industrial Research fellowship).

The project involved exposing blood samples collected from subjects to different concentrations of pesticides commonly used in India, and assessing the damage done to the cells. The techniques used were chromosomal aberrations (analysis by karyotyping), Single cell gel electrophoresis (comet assay) and micronucleus test. 


\section{Publications}

Wagner OI, Esposito A, Köhler B, Chen CW, Shen CP, Wu GH, Butkevich E, Mandalapu S, Wenzel D, Wouters FS, Klopfenstein DR. (2009) Synaptic scaffolding protein SYD-2 clusters and activates kinesin-3 UNC-104 in C. elegans. PNAS 106 (46) 19605-19610

Spilker KA. Cueva JG, Mulholland A, Mandalapu S, Klopfenstein D, Shen K, Goodman $M$. Missense mutation in mec-7/ $\beta$-tubulin affects multiple aspects of microtubule function in C. elegans mechanosensory neurons. (Under Submission) 
...... If we have no peace, it is because we have forgotten that we belong to each other

Mother Teresa 